\title{
Involvement of activation-induced cytidine deaminase in skin cancer development
}

\author{
Taichiro Nonaka, ${ }^{1,2}$ Yoshinobu Toda, ${ }^{3}$ Hiroshi Hiai, ${ }^{4}$ Munehiro Uemura, ${ }^{1}$ Motonobu Nakamura, ${ }^{5}$ Norio Yamamoto, ${ }^{6}$ Ryo Asato, ${ }^{6}$ \\ Yukari Hattori, ${ }^{7}$ Kazuhisa Bessho, ${ }^{8}$ Nagahiro Minato, ${ }^{2}$ and Kazuo Kinoshita ${ }^{1}$ \\ 'Shiga Medical Center Research Institute, Shiga, Japan. ${ }^{2}$ Department of Immunology and Cell Biology, ${ }^{3}$ Center for Anatomical Studies, and ${ }^{4}$ Laboratory for Malignancy Control Research, \\ Kyoto University Graduate School of Medicine, Sakyo-ku, Kyoto, Japan. ${ }^{5}$ Department of Dermatology, University of Occupational and Environmental Health, Kitakyushu, Japan. \\ ${ }^{6}$ Department of Otolaryngology Head and Neck Surgery, Kyoto University Graduate School of Medicine, Sakyo-ku, Kyoto, Japan. 'Department of Dermatology, Shiga Medical Center, Shiga, Japan. \\ ${ }^{8}$ Department of Oral and Maxillofacial Surgery, Kyoto University Graduate School of Medicine, Sakyo-ku, Kyoto, Japan.
}

\begin{abstract}
Most skin cancers develop as the result of UV light-induced DNA damage; however, a substantial number of cases appear to occur independently of UV damage. A causal link between UV-independent skin cancers and chronic inflammation has been suspected, although the precise mechanism underlying this association is unclear. Here, we have proposed that activationinduced cytidine deaminase (AID, encoded by AICDA) links chronic inflammation and skin cancer. We demonstrated that Tg mice expressing AID in the skin spontaneously developed skin squamous cell carcinoma with Hras and Trp53 mutations. Furthermore, genetic deletion of Aicda reduced tumor incidence in a murine model of chemical-induced skin carcinogenesis. AID was expressed in human primary keratinocytes in an inflammatory stimulus-dependent manner and was detectable in human skin cancers. Together, the results of this study indicate that inflammation-induced AID expression promotes skin cancer development independently of UV damage and suggest AID as a potential target for skin cancer therapeutics.
\end{abstract}

\section{Introduction}

Cancer development involves a cascade of genetic alterations that activate oncogenes and inactivate tumor-suppressor genes (1). Some genetic alterations are caused by external factors such as UV light, ionizing radiation, chemical mutagens, and viral infection. The mutational mechanisms in cancers associated with these environmental factors have been well studied. However, the mechanisms of somatic mutations in cancers without an apparent environmental cause remain elusive. These somatic mutations are likely caused by endogenous factors, including ROS and DNA replication errors. We previously discovered activation-induced cytidine deaminase (AID), which induces both point mutations and recombination in $\mathrm{Ig}$ genes and diversifies the $\mathrm{Ab}$ repertoire in $\mathrm{B}$ lymphocytes (2). In mice, AID overexpression caused spontaneous tumors in various organs with mutations in non-Ig genes $(3,4)$. In addition, we demonstrated that AID is expressed in human hepatocellular carcinoma (5), gastric cancer (6), and colorectal cancer (7). These observations suggest that aberrant AID expression causes mutations in various oncogenes or tumor-suppressor genes and that an accumulation of such mutations will result in cancer (8).

Most skin cancers are caused by UV light; however, some occur independently of UV light and are caused by different mutational mechanisms. For example, squamous cell carcinoma (SCC), basal cell carcinoma (BCC), and melanoma can occur on skin regions that are not commonly exposed to UV light. Clinical data suggest that chronic inflammation, chronic ulcers, and scarring are important risk factors for skin SCC in people with a darker complexion,

Conflict of interest: The authors have declared that no conflict of interest exists. Submitted: February 16, 2015; Accepted: February 4, 2016.

Reference information: J Clin Invest. 2016;126(4):1367-1382. doi:10.1172/JCI81522. although the precise molecular mechanisms are poorly understood (9). Therefore, in animal models, we examined whether AID is involved in UV-independent skin cancers. We found that AID expression is necessary for efficient skin cancer development. In addition, we demonstrated AID expression in human skin cancers (SCC, BCC, and melanoma) and head and neck cancers (SCC and melanoma) in the absence of prior UV exposure. These findings indicate that AID may be an endogenous mutagen involved in skin cancer development.

\section{Results}

AID overexpression in mice results in spontaneous skin cancer development. We reported that Tg mice with ubiquitous AID expression under the control of the chicken $\beta$-actin promoter (CAG-AID-Tg) developed both $\mathrm{T}$ cell lymphoma and lung carcinoma (3). These mice developed spontaneous melanoma, albeit at a low frequency, because of Tg AID expression in the epidermis (Figure 1A). Furthermore, immunodeficient nude CAG-AID-Tg mice (CAG-AID$\mathrm{Tg} \mathrm{nu} / \mathrm{nu}$ ) developed psoriasis-like skin lesions after skin treatment with 12-O-tetradecanoylphorbol 13-acetate (TPA) (Figure $1 \mathrm{~A}$ and Supplemental Figure 1; supplemental material available online with this article; doi:10.1172/JCI81522DS1). These findings suggest that aberrant AID expression is associated with cell proliferation-related skin lesions, such as hyperplasia, cancer, and psoriasis. Long-term observation of CAG-AID-Tg mice was impossible, because they developed $\mathrm{T}$ cell lymphoma and typically died within 40 weeks of birth. To resolve this issue, we generated a new Tg line of mice, K14-AID-Tg, using a construct in which the Aicda gene was placed under the control of the epidermal basal cell-specific keratin 14 promoter (Figure 1B). We selected the $\mathrm{FVB} / \mathrm{N}$ strain as the genetic background, as this strain is highly 


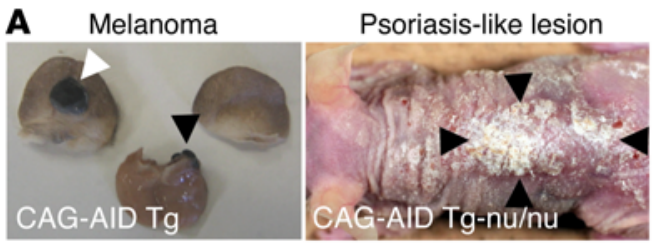

B

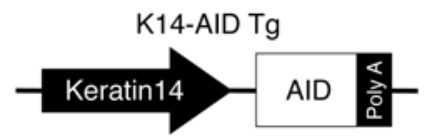

FVB/N, Ptch1 T1267N

G

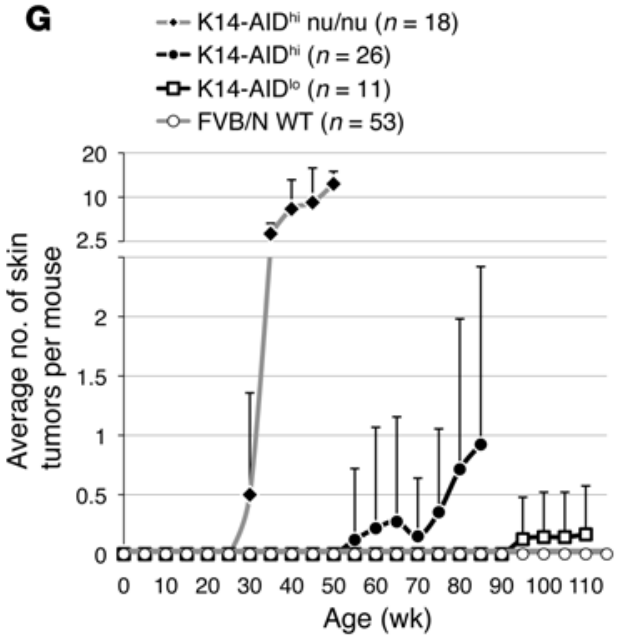

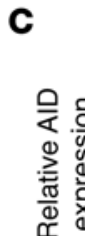

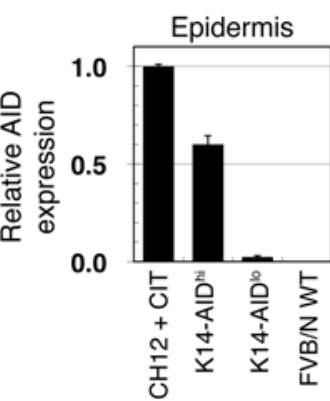

D

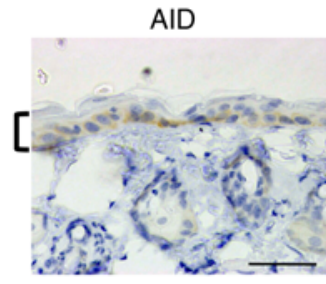

H

$-\bullet-\mathrm{K} 14-\mathrm{AID} \mathrm{D}^{\mathrm{hi}} \mathrm{nu} / \mathrm{nu}(n=18)$

-o- K14-AID ${ }^{\text {hi }}(n=26)$

$-\square-\mathrm{K}_{14}-\mathrm{AID}^{10}(n=11)$

$-\infty$ FVB/N WT $(n=53)$

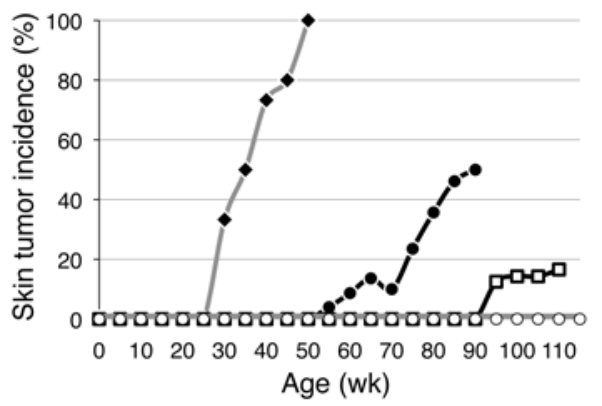

E
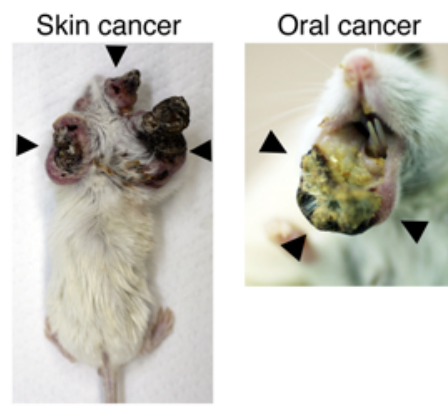

$\mathbf{F}$

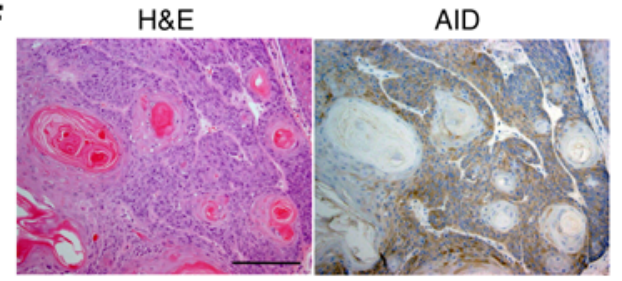

Figure 1. Spontaneous skin carcinogenesis in AID-Tg mice. (A) Representative images of spontaneously developed melanomas in the auricles of CAGAID-Tg mice and psoriasis-like skin lesions in TPA-treated CAG-AID-Tg nu/nu mice. (B) Generation of keratin 14 promoter-driven AID-Tg mice on an FVB/N (homozygous Ptch1 T1267N) background. (C) Relative Aicda mRNA expression in K14-AID-Tg epidermis (mean \pm SD, $n=3$ ). K14-AID hi and K14-AID ${ }^{10}$ indicate mice with high and low K14-AID transgene expression, respectively. (D) Anti-AID staining of the epidermis from K14-AID ${ }^{\text {hi }}$ mice. Bracket indicates the epidermis. Scale bar: $100 \mu \mathrm{m}$. (E) Representative images of spontaneous skin and oral cancers on K14-AID hi mice. (F) H\&E and anti-AID staining of spon-

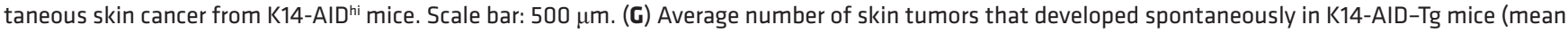
\pm SD). All mouse groups had homozygous Ptch1 T1267N alleles. nu/nu, mice with homozygous nude alleles. (H) Incidence of skin tumors that developed spontaneously in K14-AID-Tg mice. (I) Kaplan-Meier survival curves for K14-AID-Tg mice. ${ }^{*} P<0.0001$, by log-rank test.

susceptible to H-Ras-induced SCC because of a T1267N amino acid polymorphism in Ptch1 (10). We confirmed AID expression at the transcriptional and protein levels using quantitative reverse transcription PCR (qRT-PCR) and IHC, respectively, and selected 2 lines with relatively high and low AID expression (K14-AID ${ }^{\text {hi }}$ and K14-AID ${ }^{\text {lo; }}$ Figure 1, C and D).

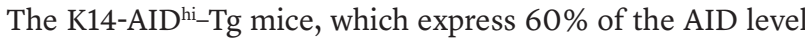
detected in a mouse B lymphoma cell line ( $\mathrm{CH} 12)$ stimulated to undergo Ig gene recombination using CD40 ligand, IL-4, and TGF- $\beta$ (CIT) (11), spontaneously developed tumors of the skin and oral mucosa at approximately 50 weeks of age, which reflects the specificity of keratin 14 promoter activity (Figure 1E). Approximately $42 \%$ of skin tumors arose on the skin in the head and neck area (head, face, and ear), and the remaining tumors arose on the skin of the trunk and limbs (Supplemental Table 1). On the basis of a pathological evaluation, the mice were diagnosed with SCC, and immunohistochemical analysis revealed that cancer cells strongly expressed AID (Figure 1F). K14-AID ${ }^{\text {lo }}$ mice, which express only
$2 \%$ of the AID observed in CIT-stimulated CH12, spontaneously developed SCC at approximately 90 weeks of age, but at a lower tumor incidence than that observed in K14-AID ${ }^{\text {hi }}-\mathrm{Tg}$ mice (Figure $1, \mathrm{G}$ and $\mathrm{H}$ ). Introduction of the nude mouse genetic background into the K14-AID ${ }^{\text {hi }}$-Tg line remarkably accelerated tumor onset and increased the number of skin tumors (Figure 1, G and H, and Supplemental Figure 2). Kaplan-Meier survival curves showed a significant decrease in the survival rate of the K14-AID ${ }^{\text {hi }}$-Tg line compared with the K14-AID ${ }^{\mathrm{lo}}$-Tg line, whereas no difference was observed between the K14-AID ${ }^{\text {lo }}$ and WT mice (Figure 1I).

AID initiates, promotes, and progresses skin tumors in a chemically induced skin carcinogenesis model. An experimental mouse skin carcinogenesis model indicated that carcinogenesis is a multistep process with at least 3 major stages (12). The first stage is initiation, which involves the mutagenic events of carcinogens. The second stage is promotion, which is induced by agents that promote clonal expansion of initiated cells. Chronic treatment of initiated skin with promoting agents results in benign squamous 
A

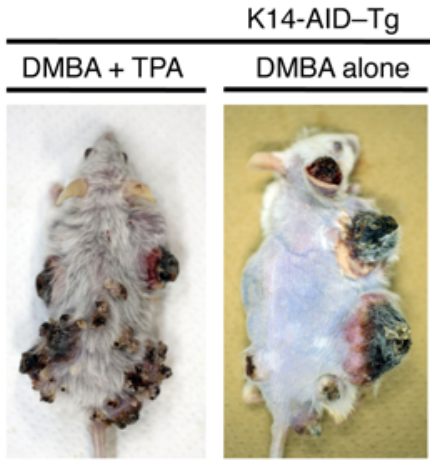

B

Classical model

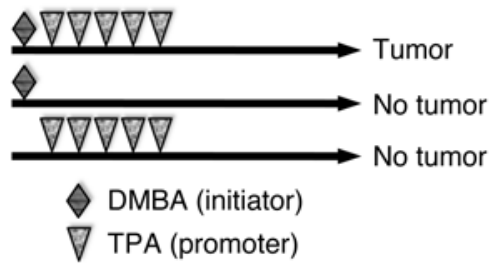

D

$\mathrm{DMBA}+\mathrm{TPA}$

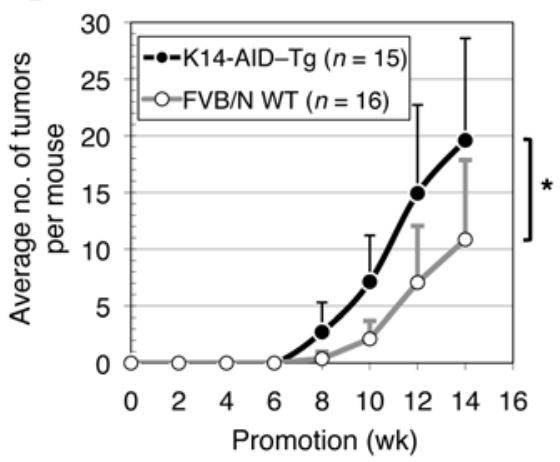

G

DMBA + TPA

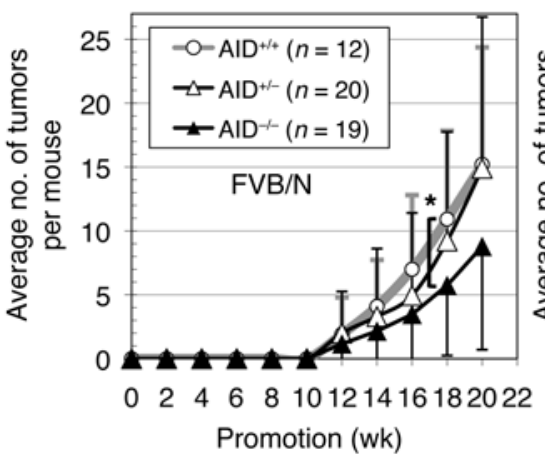

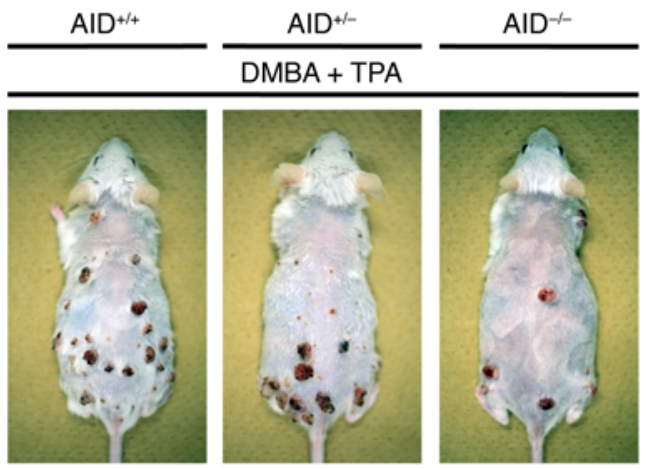

$\mathrm{DMBA}+\mathrm{TPA}$
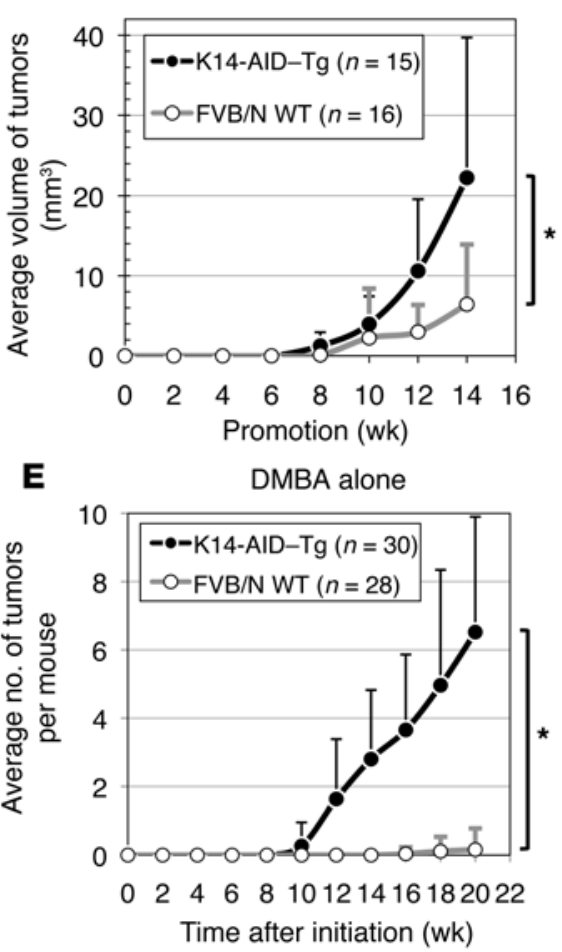

$\mathbf{F}$

TPA alone

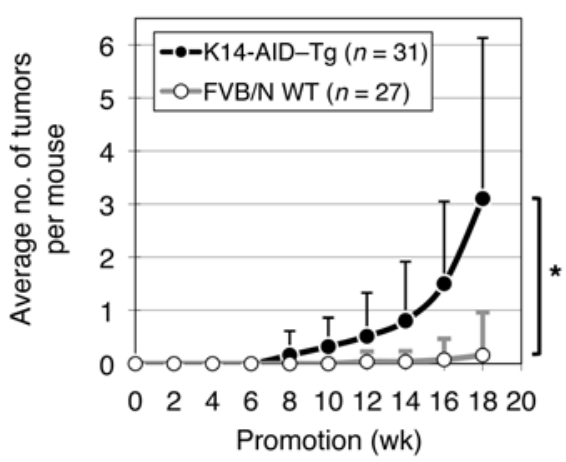

I

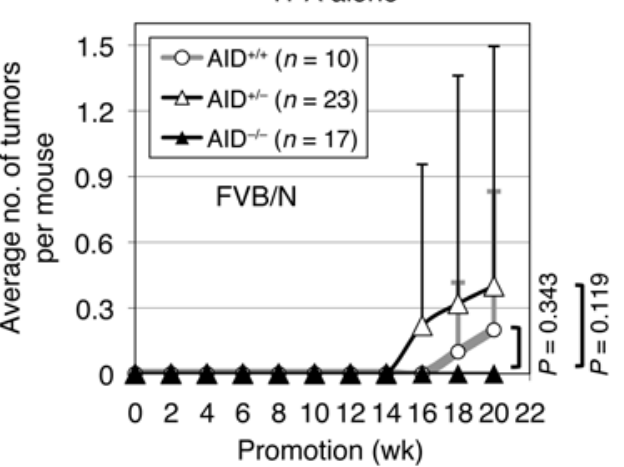

DMBA + TPA

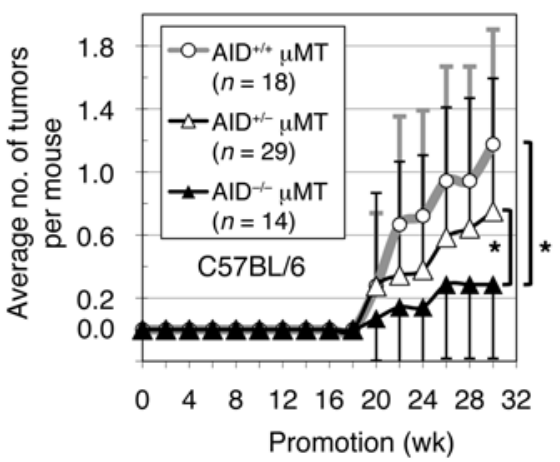

Figure 2. Initiation and promotion of skin tumors by aberrant AID expression in a chemically induced skin carcinogenesis model. (A) Representative photographs of skin tumors in mice. For the DMBA-plus-TPA groups, a single 260-nmol dose of DMBA was applied to the dorsal skin of the mice, and 2 weeks after initiation, $16 \mathrm{nmol}$ TPA was applied twice weekly for the duration of the experiments. For the DMBA-alone group, a single 260-nmol dose of DMBA was applied. For the TPA-alone group, $16 \mathrm{nmol}$ TPA was applied twice weekly for the duration of the experiment. Each photograph was taken 20 weeks after TPA treatment, except the photograph of the K14-AID-Tg mice treated with DMBA (22 weeks) or TPA alone (29 weeks). (B) Schematic representation of multistep chemically induced skin carcinogenesis. Skin tumors develop following single DMBA exposure and both subsequent and multiple TPA exposures. (C) Average volume of skin tumors in K14-AID-Tg mice treated with DMBA and TPA (mean \pm SD). ${ }^{*} P<0.05$, by Welch's $t$ test. (D-I) Average number of skin tumors that developed over time in mice treated with DMBA and/or TPA (mean \pm SD). AID ${ }^{+/+}$, WT; AID ${ }^{+/-}$, heterozygote; AID ${ }^{-/-}$, deficient mice. ${ }^{*} P<0.05$, by Welch's $t$ test. 
Table 1. Malignant conversion of chemically induced skin tumors in K14-AID-Tg mice

\section{Total no. of tumors in mice}

Mice

K14-AID $(n=25)$

K14-AID ${ }^{\text {hi }}(n=14)$

FVB/N WT ( $n=13)$

Treatment
DMBA alone
DMBA + TPA
DMBA + TPA

$\mathrm{SCC}$

88

65

5

Papilloma
75
296
190

190

\section{No. of tumors per mouse}

SCC Papilloma

$3.52 \quad 3.00$

$4.64 \quad 21.14$

$0.38 \quad 14.62$

\section{$\%$ of SCC \\ $53.99^{\mathrm{B}}$ \\ $18.01^{\mathrm{A}}$ \\ 2.56}

Mice in the DMBA-alone group were treated with a single 260-nmol dose of DMBA. For the DMBA-plus-TPA groups, at 2 weeks after initiation with DMBA, $16 \mathrm{nmol}$ TPA was applied twice weekly for 18 weeks. All mice were observed at 20 weeks after DMBA treatment. No., number. ${ }^{A} P<0.0001$ for K14-AID ${ }^{\mathrm{hi}}$ $(\mathrm{DMBA}+\mathrm{TPA})$ versus FVB/N WT (DMBA + TPA); ${ }^{\mathrm{B}} P<0.0001$ for K14-AID ${ }^{\text {hi }}$ (DMBA alone) versus K14-AID ${ }^{\text {hi }}(\mathrm{DMBA}+$ TPA), by Fisher's exact test.

papilloma development. The third stage is progression, in which benign tumors progress to malignant SCC spontaneously or following additional carcinogenic treatments. To determine the stage in which AID participates, we treated K14-AID ${ }^{\text {hi }}$ mice with 7,12-dimethylbenz[a] anthracene (DMBA) as an initiator and TPA as a promoter. In this model, after DMBA introduces a gain-offunction mutation in Hras1 codon 61, TPA induces cell growth via the PKC-signaling pathway and, eventually, skin tumor formation (13). However, DMBA or TPA treatment alone failed to induce tumors in WT mice (Figure 2B). In our experiment, compared with treated WT mice, K14-AID ${ }^{\text {hi }}$ mice treated with DMBA and TPA showed a significant increase in the average volume and number of tumors (Figure 2, A, C, and D). Surprisingly, treatment with DMBA or TPA alone induced tumors in K14-AID ${ }^{\text {hi }}$ mice (Figure 2, $A, E$, and F). These findings suggest that AID can partly substitute for DMBA or TPA in the carcinogenesis process and plays roles in the initiation and promotion of skin tumors.

The overall malignant conversion frequency was 7-fold higher in $\mathrm{K}_{14}-\mathrm{AID}^{\text {hi }}$ mice than in WT mice following treatment with both DMBA and TPA (18\% versus 2.6\%, $P<0.0001$, Table 1$) . K_{14}-A^{\text {hi }}$ skin tumors arising from DMBA alone had a 3-fold higher malignant conversion frequency than did K14-AID ${ }^{\text {hi }}$ tumors induced by both DMBA and TPA ( $54 \%$ versus $18 \%, P<0.0001$, Table 1 ). When SCC and papilloma were separately counted 20 weeks after DMBA treatment followed by repetitive TPA administration, the number of papillomas per mouse was moderately higher in K14-AID ${ }^{\text {hi }}$ mice than in WT mice (21 versus 15, Table 1), whereas the number of SCCs per mouse increased significantly in K14-AID ${ }^{\text {hi }}$ mice (4.6 versus 0.38, Table 1). Compared with the DMBA-plus-TPA protocol, DMBA treatment alone resulted in a decreased number of papillomas in K14-AID ${ }^{\text {hi }}$ mice (from 21 to 3.0, Table 1), whereas the number of SCCs only slightly decreased (from 4.6 to 3.5, Table 1). These findings suggest that the K14-AID transgene is a potent driver of malignant conversion and a mild tumor promoter.

To determine whether endogenous AID contributes to chemically induced skin carcinogenesis, we treated AID-deficient mice (2) with DMBA and TPA. Because C57BL/6 mice are resistant to chemically induced skin carcinogenesis, we used AID-deficient mice on an FVB/N background, which harbor homozygous Ptch1 T1267N alleles. We found that when compared with WT mice, AID-defi-
Figure 3. Promotion of oral tumors by aberrant AID expression in a 4NQO-induced oral carcinogenesis model. (A) Representative photographs of oral tumors developed in K14-AID-Tg mice after application of 4 NQO to the oral mucosa. In mice, oral mucosa was treated with $20 \mu \mathrm{l}$ 4NQO $(5 \mathrm{mg} / \mathrm{ml})$ thrice weekly for the duration of the experiment. (B) Average number and frequency of oral tumors arising over time in K14-AID-Tg mice treated with $4 \mathrm{NQO}$ (mean $\pm \mathrm{SD}$ ). ${ }^{*} P<0.05$, by Welch's $t$ test.

A

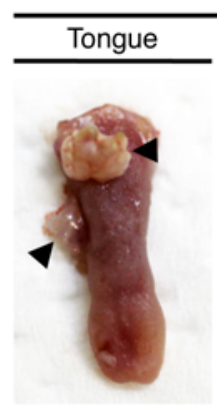

B

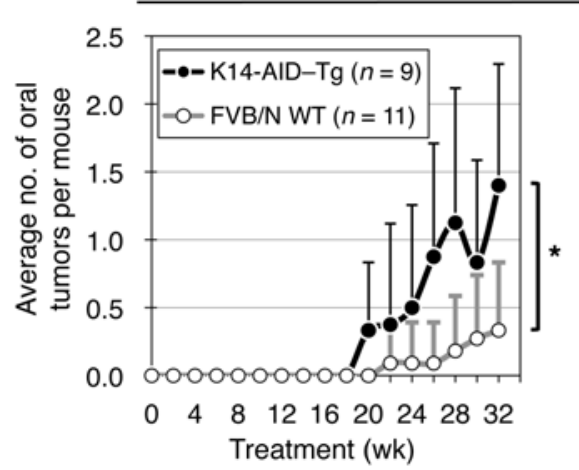

$\mathrm{K} 14-\mathrm{AID}-\mathrm{Tg}+4 \mathrm{NQO}$

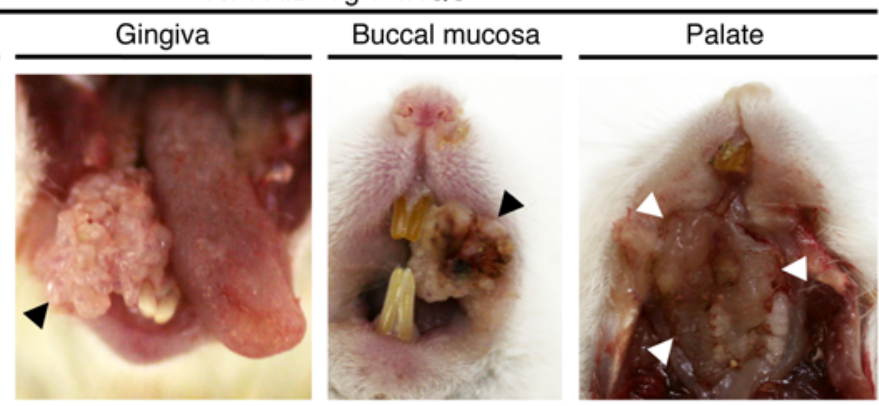

$4 \mathrm{NQO}$

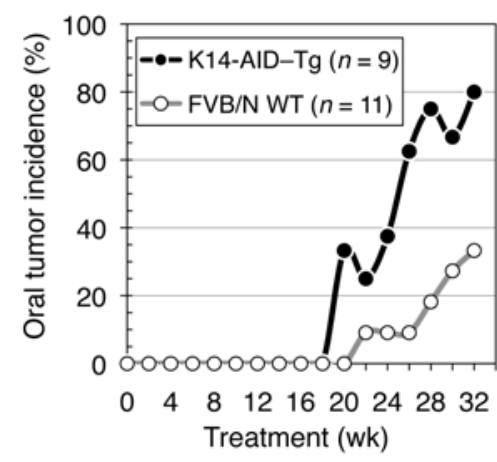


Table 2. Hras1 and Trp53 gene mutation frequency in skin tumors from AID-Tg mice

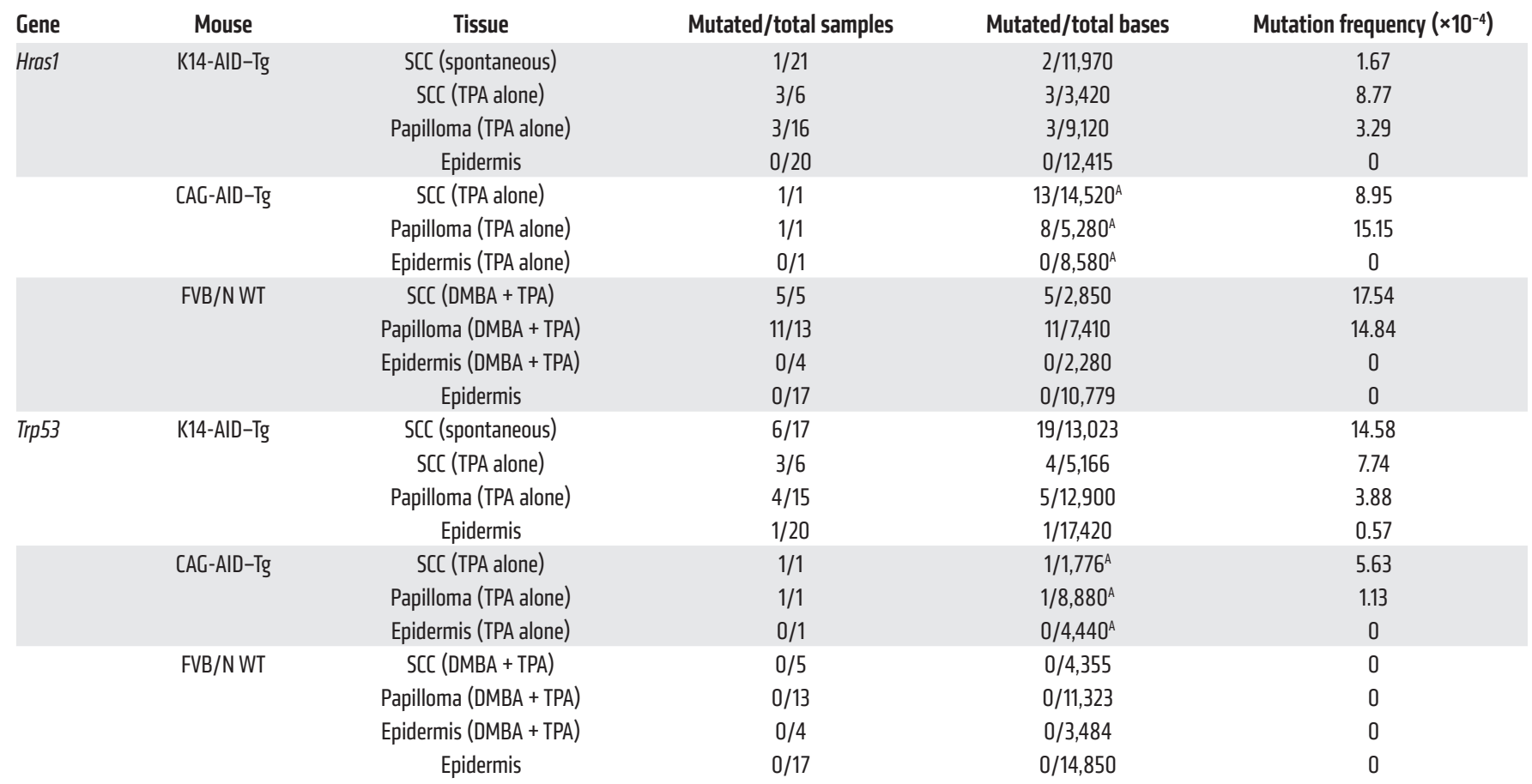

APCR product was cloned into the vector and sequenced. A detailed list of mutations is provided in Supplemental Table 2.

cient mice treated for at least 18 weeks with both DMBA and TPA exhibited a significantly lower average number of tumors (Figure 2, A and G). When treated with TPA alone, the AID-deficient mice often had a lower tumor incidence than did WT and heterozygous mice, although this difference was not significant (Figure $2 \mathrm{H}$ ).

Previously, IgG-induced FcR $\gamma$ activation in the microenvironment was reported to be important for skin cancer development (14). Because IgG is not produced in AID-deficient mice, our findings may reflect a lack of IgG rather than a lack of AID expression in keratinocytes. To examine this possibility, we introduced the AID-deficient allele into Ig-deficient mice through cross-breeding with $\mu \mathrm{MT}$ mice on a C57BL/6 background (15). The resulting AID-deficient $\mu \mathrm{MT}$ mice (AID ${ }^{-/} \mu \mathrm{MT}$ ) exhibited a significantly lower tumor incidence following treatment with DMBA and TPA compared with AID-sufficient $\mu \mathrm{MT}$ mice ( $\mathrm{AID}^{+/+} \mu \mathrm{MT}$ ), although the skin tumor-resistant C57BL/6 background reduced the overall tumor burden (Figure 2I). These results indicate that endogenous AID participates in chemically induced skin carcinogenesis.

To determine whether AID contributes to squamous cell tumor formation in a different tumor model, we examined a chemically induced oral carcinogenesis model developed using 4-nitroquinoline 1-oxide (4NQO). Similar to some carcinogens found in tobacco, continuous application of $4 \mathrm{NQO}$ to the oral mucosa of mice results in the formation of DNA adducts that cause mutations, eventually resulting in oral cancer (16). Because the histological type of 4NQOinduced oral cancer is similar to that of human oral cancers, this model has been used to study tobacco-induced oral carcinogenesis (17). When applied to the oral mucosa of K14-AID ${ }^{\text {hi }}$ mice, $4 \mathrm{NQO}$ induced squamous papilloma and SCC development throughout the oral mucosa, including the tongue, gingiva, buccal mucosa, and palate (Figure $3 \mathrm{~A})$. The number and incidence of oral tumors were significantly higher in K14-AID ${ }^{\text {hi }}$ mice than in WT mice (Figure 3B).

AID introduces gain-of-function mutations in Hras1 and loss-offunction mutations in Trp53. We identified genetic mutations introduced into the Hras1 and Trp53 genes in skin tumors from AID-Tg mice that developed SCC spontaneously or with TPA treatment alone (Table 2 and Supplemental Table 2). Hras1 mutations were concentrated around $\mathrm{G}$ domains such as G13I (corresponding to the base substitutions c.37G $>\mathrm{A}$ and c.38G $>\mathrm{T}$ ); G13V (c.38G $>\mathrm{T}$ ); and Q61L (c.182A>T) (Figure 4, A and B, and Supplemental Table 2). These mutations convert $\mathrm{H}$-Ras to a constitutively active form by inhibiting GTP hydrolysis, which subsequently initiates carcinogenesis $(18,19)$. In addition, the Hras 1 mutation frequency was higher in mice that developed SCC with TPA treatment alone than in those that developed SCC spontaneously, suggesting a stronger dependence on Hras1 activation in TPA-induced SCC development in AID-Tg mice (Table 2).

In more than $90 \%$ of human skin SCC cases, p53 mutations are caused by UV exposure, and TP53 mutations are more common in the DNA-binding domain $(20,21)$. Trp53 mutation analysis in K14AID-Tg mice with spontaneous SCC identified several mutations with amino acid substitutions in the DNA-binding domain (Figure 4C and Supplemental Table 2). Several mutated Trp53 alleles detected in the K14-AID-Tg mice were predicted to express functionally compromised $\mathrm{p} 53$ proteins on the basis of the International Agency for Research on Cancer (IARC) TP53 database (ref. 22 and Supplemental Table 2). TP53 hot-spot mutations found in codons $247,248,278$, and 281 in human skin SCC were not identified in K14- 
A

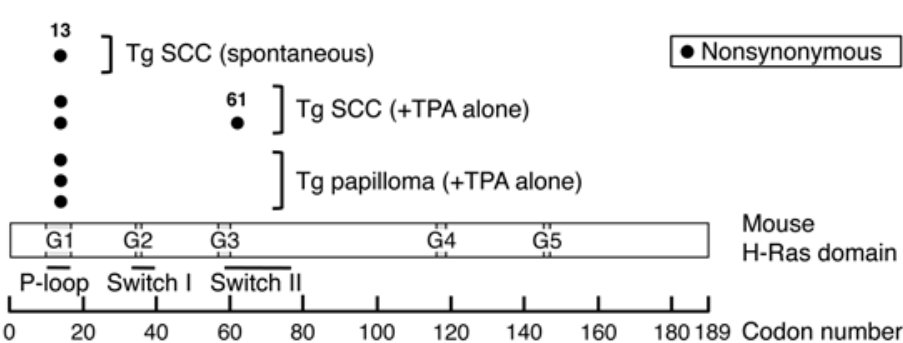

C

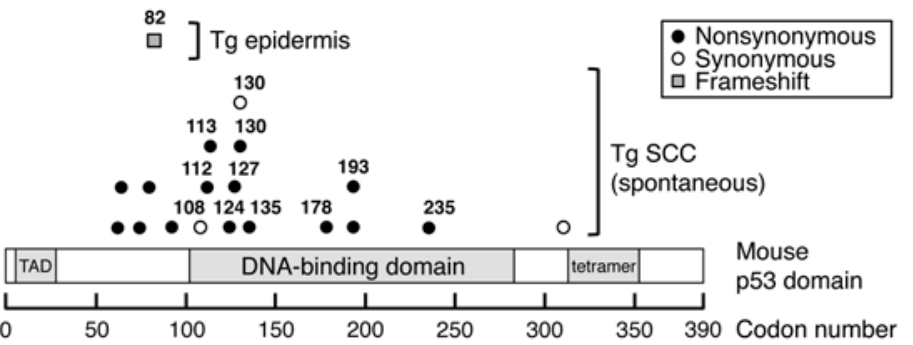

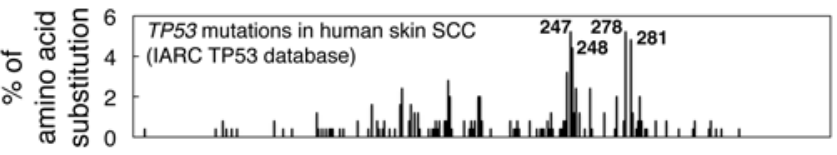

E
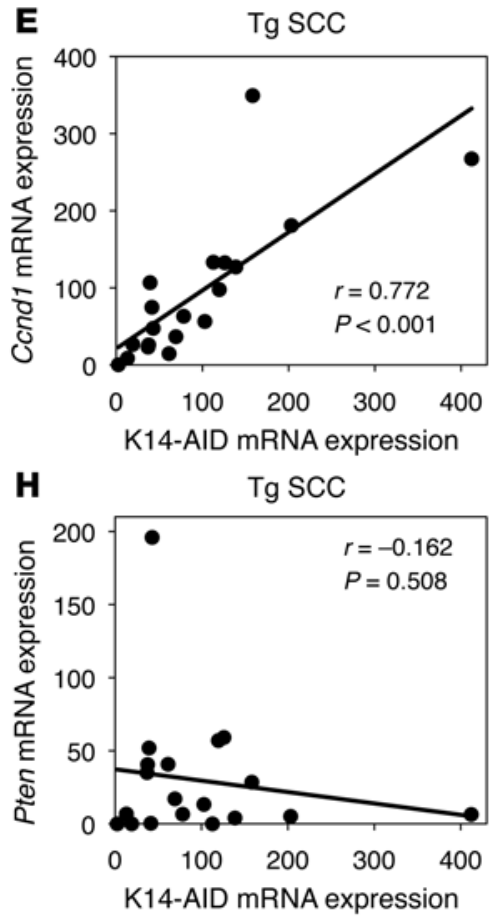

F $\quad \mathrm{Tg} \mathrm{SCC}$

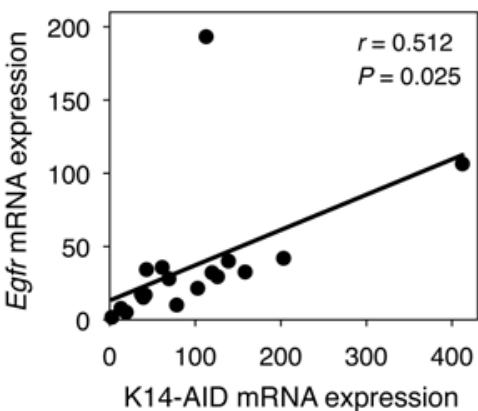

I

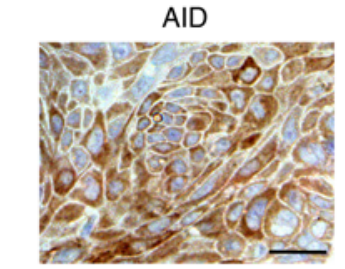

Phospho-ERK1/2

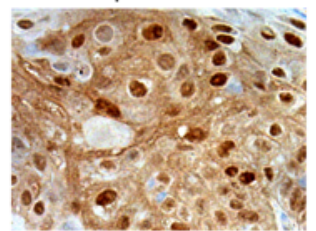

B

\begin{tabular}{|c|c|c|c|c|c|c|}
\hline \multirow{2}{*}{\multicolumn{2}{|c|}{\begin{tabular}{|c|}
$\mathrm{Tg}, \mathrm{SCC}$ \\
Hras1
\end{tabular}}} & & \multirow[b]{2}{*}{ Total } \\
\hline & & A & $\mathrm{T}$ & G & & \\
\hline \multirow[t]{4}{*}{ From } & $n$ A & 1 & 1 & 0 & 0 & 1 \\
\hline & $T$ & 0 & 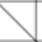 & 0 & 0 & 0 \\
\hline & G & 1 & 3 & 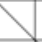 & 0 & 4 \\
\hline & C & 0 & 0 & 0 & $\searrow$ & 0 \\
\hline & Total & 1 & 4 & 0 & 0 & 5 \\
\hline
\end{tabular}

D

\begin{tabular}{|l|c|c|c|c|c|c|}
\hline \multicolumn{2}{|c|}{$\begin{array}{l}\text { Tg, SCC } \\
\text { Trp53 }\end{array}$} & To & T & G & C & Total \\
\hline \multirow{2}{*}{ From } & A & & 1 & 0 & 0 & 1 \\
\cline { 2 - 6 } & T & 0 & & 0 & 1 & 1 \\
\hline G & 2 & 2 & & 0 & 4 \\
\hline C & 3 & 8 & 2 & & 13 \\
\hline \multicolumn{2}{|c|}{ Total } & 5 & 11 & 2 & 1 & 19 \\
\hline
\end{tabular}

Trp53 nucleotide 5'-flanking C-to-T

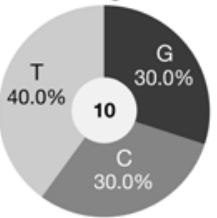

G

$\operatorname{Tg~SCC}$

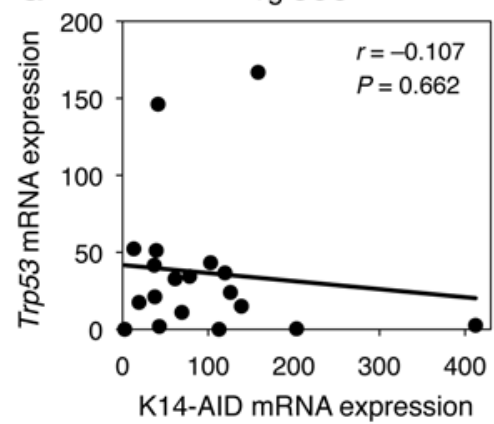

K14-AID mRNA expression

Cyclin D1

EGFR

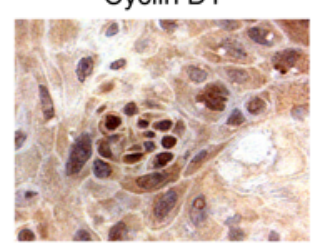

Phospho-AKT

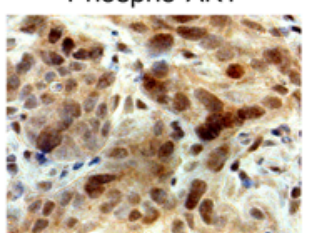

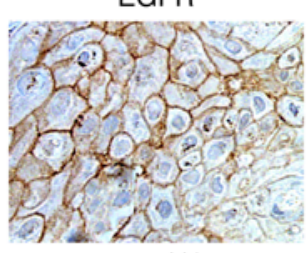

p16 $6^{\operatorname{lnk} 4 a}$

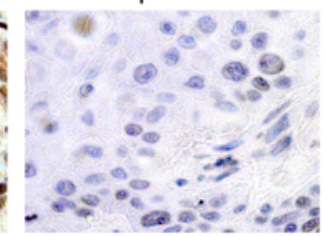

Figure 4. Molecular characterization of spontaneous SCC in K14-AID-Tg mice. (A) Codon distribution of Hras1 somatic mutations. Black circles indicate nonsynonymous mutations. The number above each black circle indicates the codon number. (B) Base substitution patterns identified in Hras1 in spontaneous and TPA-induced SCC. (C) Codon distribution of Trp53 somatic mutations. Black and white circles and gray rectangles indicate nonsynonymous, synonymous, and frameshift mutations, respectively. Frequency of TP53 somatic mutations in human skin SCC from the IARC TP53 database juxtaposed for comparison with distributions and hot spots seen in K14-AID-Tg mice. The top 4 codon numbers of mutations in human skin SCC are shown. (D) Base substitution patterns and frequency of the nucleotide 5 '-flanking C-to-T transition in Trp53 in spontaneously developed SCC. The number in the center of the pie chart is the sum of the C-to-T and G-to-A changes. (E-H) Correlations of transcript levels between the K14-AID transgene and Ccnd1, Egfr, Trp53, and Pten, respectively, in spontaneous SCC. Pearson's correlation statistic was used to measure the extent of the relationships between the expression levels of 2 genes. In A-D, data points indicate the individual K14-AID-Tg mice listed in Supplemental Table 2. (I) IHC of spontaneous SCC developed in K14AID-Tg mice. Scale bar: $20 \mu \mathrm{m}$. 
A
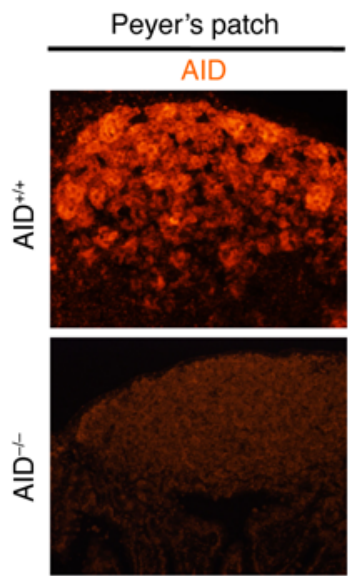

B
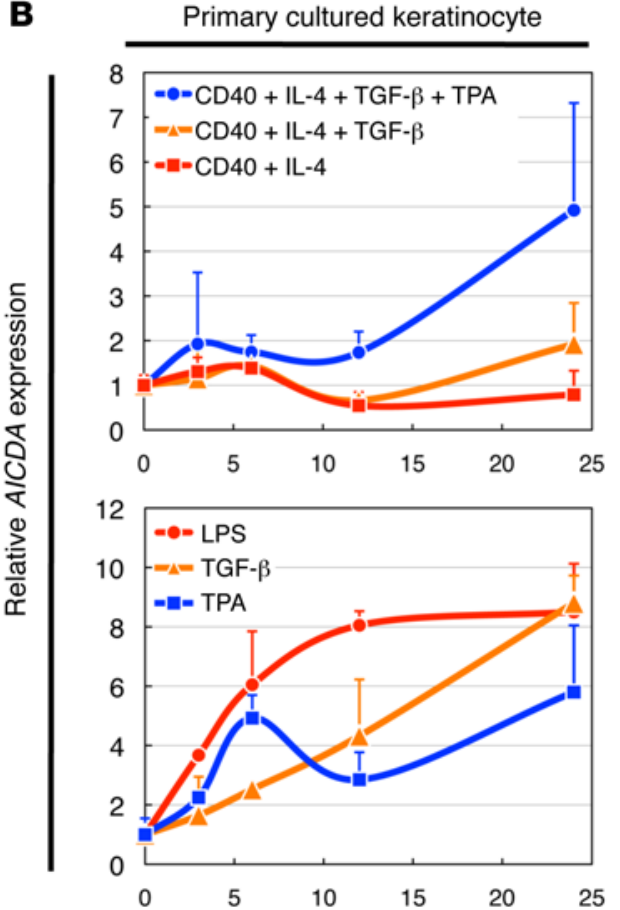
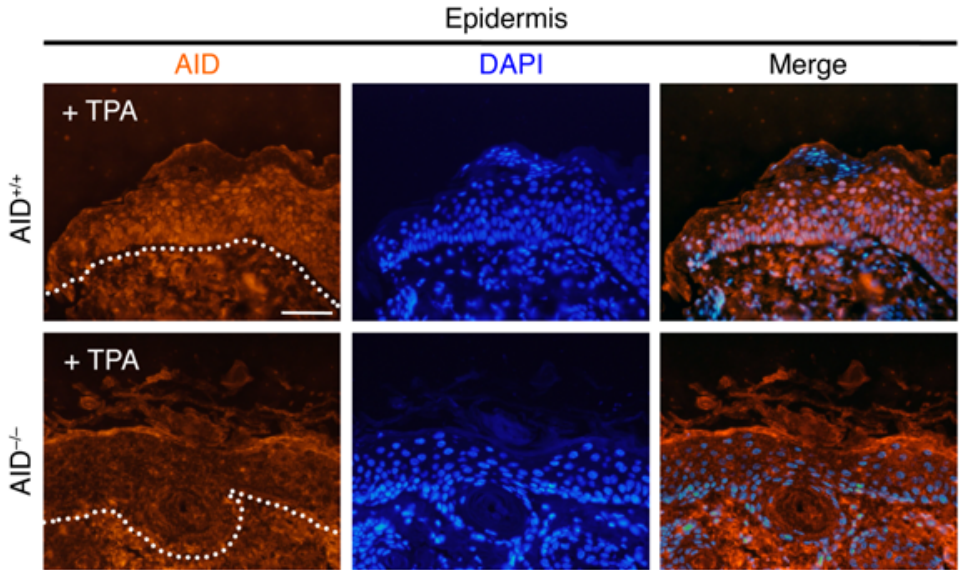
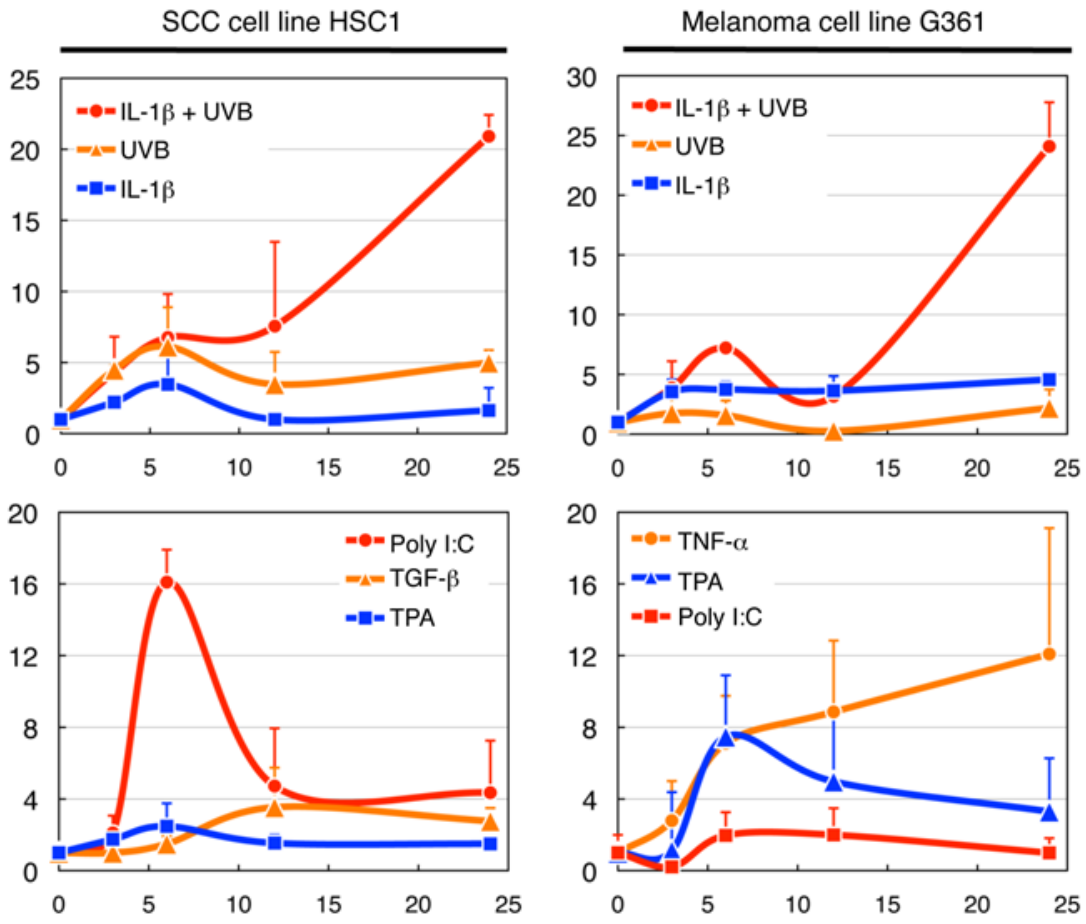

Time after stimulation (h)

Figure 5. Stimulus-dependent endogenous AID expression in keratinocytes. (A) Fluorescent immunostaining of AID in Peyer's patch and epidermis from mice. Mice were treated with $16 \mathrm{nmol}$ TPA twice weekly for a total of 4 applications. Skin tissue was harvested 48 hours after the last treatment. Dashed line indicates the basal layer. Orange, AID; blue, DAPI counterstain of DNA. Scale bar: $100 \mu \mathrm{m}$. (B) Fold change of relative AICDA mRNA expression in human keratinocytes, an SCC cell line, and a melanoma cell line after various stimulations (mean $\pm S D, n=3$ ).

AID-Tg mice (Figure 4C). Because mutations in codons 247, 248, and 278 are considered to be introduced by the UV light-induced pyrimidine dimer formation of CC $>$ TT (UV signature mutation: $\geq 60 \%$ are $\mathrm{C}>\mathrm{T}$ at the dipyrimidine site, with $\geq 5 \%$ being $\mathrm{CC}>\mathrm{TT}$ ) (23), the absence of these mutations may be expected in K14-AIDTg mice. None of the Trp53 mutations previously identified in UV- or DMBA-induced murine skin tumors were observed in K14-AID-Tg mice $(24,25)$. The most common base substitution observed in K14AID-Tg mice was a C-to-T transition or G-to-A transition on the opposite strand (Figure 4D). This pattern is the preferential footprint of cytidine deaminases such as AID and apolipoprotein B mRNAediting enzyme catalytic polypeptide-like 3 (APOBEC3). This pattern is distinct from DMBA-induced mutations, in which A-to-T transversion is dominant ( $>60 \%)$ (25). This tendency was observed in Trp53 mutations of DMBA-plus-TPA-induced murine skin SCC (ref. 25 and Supplemental Tables 3 and 4). The AID mutation signature is considered a C-to-T transition preceded by G or A (26), whereas a C-to-T transition preceded by $\mathrm{C}$ or $\mathrm{T}$ is characteristic of APOBEC3 mutation (27). Among the 10 observed C-to-T transitions in the tumor Trp53 sequences, $3(30 \%)$ mutations were 5 '-flanked by $\mathrm{G}$ (Figure 4D). In addition, we examined mutations in AID-Tg mice with TPA-induced SCC using a different promoter (CAG-AID-Tg) and identified a Q61L mutation in Hras1 and a frameshift mutation in Trp53 (Supplemental Table 2). These results suggest that AID overexpression contributes to Hras 1 and Trp53 mutagenesis, which is responsible for skin tumor development. 
A
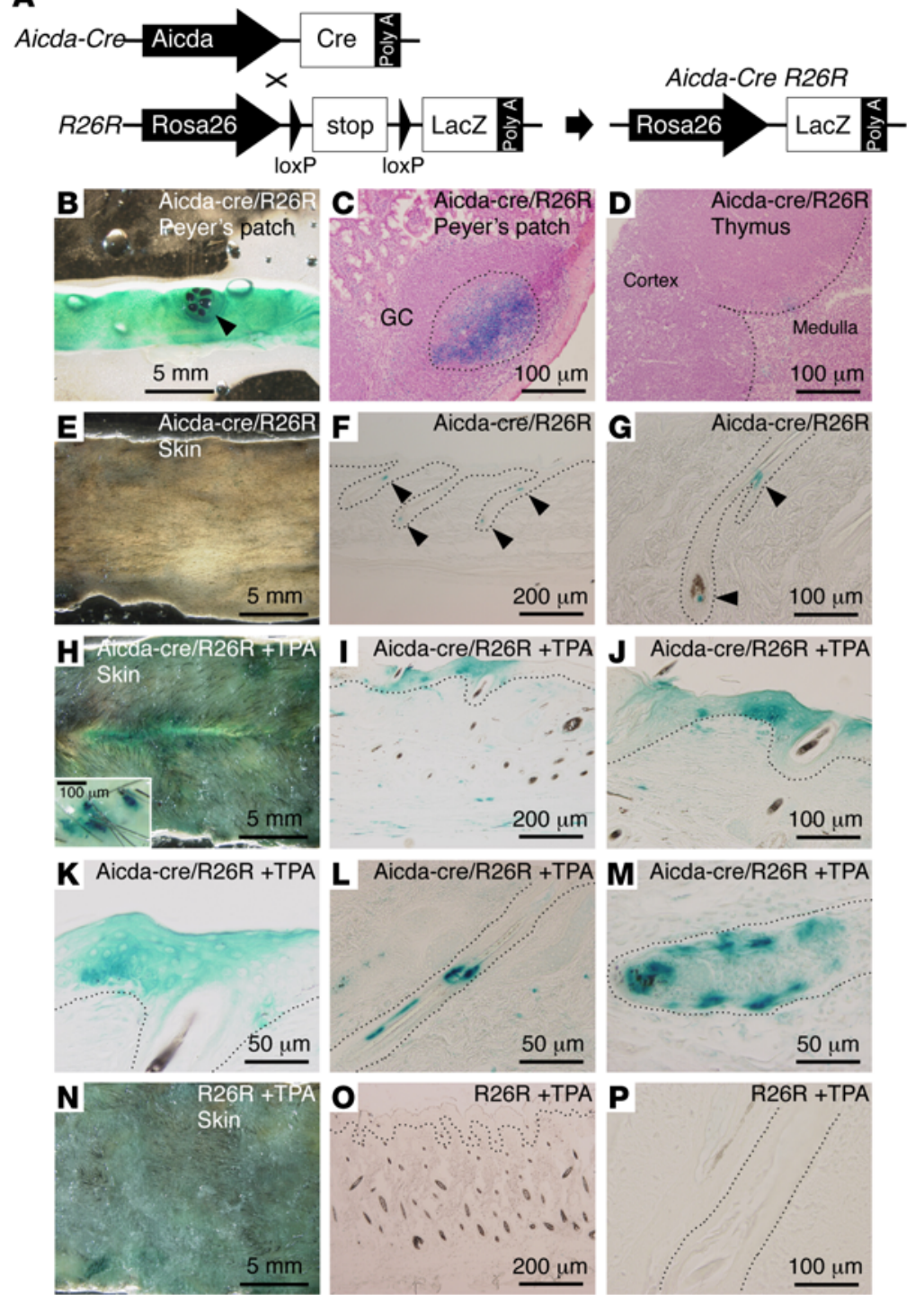

Figure 6. AID fate mapping in TPA-treated skin from Aicda-Cre R26R double-Tg mice. (A) Schematic representation of the Aicda-Cre and R26R alleles used for AID fate mapping. Cre-mediated excision of a loxP-flanked transcriptional stop induces lac Z expression. (B) Macroscopic image of a Peyer's patch from Aicda-Cre R26R double-Tg mice after lacZ staining. Arrowhead indicates lacZ activity in the Peyer's patch. (C and $\mathbf{D})$ LacZ and $\mathrm{H} \& \mathrm{E}$ double staining of a Peyer's patch (C) and thymus (D) from Aicda-Cre R26R mice. Dashed line indicates the germinal center (C) and the cortical-medullary boundary (D). GC, germinal center. (E-C) LacZ staining of skin from Aicda-Cre R26R mice. Dashed line indicates the basal layer; arrowheads indicate lac $Z$ activity in hair bulbs and root sheaths near sebaceous glands. (H-P) Dorsal skin from Aicda-Cre R26R (H-M) and R26R single-Tg (N-P) mice was treated with $16 \mathrm{nmol}$ TPA twice weekly for 6 weeks, and the skin was subsequently analyzed for lacZ activity. Dashed line indicates the basal layer. $(\mathbf{E}, \mathbf{H}$, and $\mathbf{N})$ Macroscopic images of skin after lacZ staining. (F, I, and $\mathbf{O})$ Entire skin layer; (J and $\mathbf{K}$ ) interfollicular epidermis; (G, L, and $\mathbf{P})$ hair follicle; and (M) hair bulb.

sion of the K14-AID transgene (Figure 4, E and F). In contrast, expression of the tumor-suppressor genes Trp53 and Pten did not correlate with that of the K14AID transgene (Figure 4, G and H). Using immunohistochemical analysis, we confirmed strong cyclin D1 and EGFR expression and accumulation of phosphorylated ERK1/2 (Thr202/Tyr204) and AKT (Ser473) in the nuclei of SCC cells developed spontaneously in K14-AID-Tg mice, thus indicating the activation of MAPK and AKT signaling (Figure 4I and Supplemental Figure 3). Therefore, similar to human SCC cases, K14AID-Tg mice with spontaneous SCC developed highly proliferative tumors.

The cyclin-dependent kinase inhibitor $\mathrm{p} 16^{\mathrm{INK} 4 \mathrm{a}}$ induces senescence in human cells and is often inactivated in human cancers, including SCC $(29,30)$. We recently reported that aberrant AID activity in human and mouse gastric cells introduced genetic mutations into the $C D K N 2 A$ and $C d k n 2 a$ genes and reduced the copy number at the CDKN2A locus (31). As observed in a gastric cancer model, immunohistochemical analysis of spontaneous SCC from K14-AID-Tg mice failed to detect $\mathrm{p} 16^{\text {Ink4a }}$, suggesting that $\mathrm{p} 16$ inactivation may be a common feature of both human and mouse SCC (Figure 4I and Supplemental Figure 3). Further investigation is needed to determine whether AID is involved in $\mathrm{p} 16^{\mathrm{INK} 4 \mathrm{a} / \mathrm{Ink} 4 \mathrm{a}}$ inactivation in skin SCC.

AID is induced in keratinocytes in an inflammatory stimulus- and UV-dependent manner. Although AID is physiologically expressed in B cells, its expression level is low in epithelial cells. To determine whether endogenous AID expression is induced in the epidermis, we used IHC to evaluate AID expression after applying TPA to the skin of $\mathrm{FVB} / \mathrm{N}$ mice. We observed that TPA treatment induced endogenous AID expression (Figure 5A). Furthermore, we analyzed TPA-induced AID expression levels using qRT-PCR and found that relative AID expression in TPA-treated epidermis was approximately $5 \%$ of that in CIT-stimulated $\mathrm{CH} 12$ cells and $9 \%$ of that

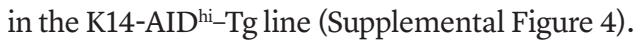

To investigate whether AID expression is induced in cultured keratinocytes, we stimulated human primary cultured keratinocytes with various agents and analyzed them using qRT-PCR. Following our previous report that AID expression is increased in mouse B cells in the presence of CIT (11), we treated the keratinocytes with CIT and observed oscillations in AICDA expression (Figure 5B). AICDA expression was further enhanced by cotreatment with TPA and CIT and was

SCC in AID-Tg mice shares common molecular characteristics with human SCC. In various human SCC cases, EGFR is hyperactive and activates RAS, which subsequently activates MAPK signaling (28). We investigated whether MAPK signaling or the alternative AKT pathway was activated in AID-induced SCC by analyzing signal transduction in K14-AID-Tg mice with spontaneous SCC. A transcript analysis revealed that mRNA expression of the tumor-promoting genes Ccnd1 and Egfr correlated with expres- induced by a single-agent treatment with LPS, TGF- $\beta$, or TPA. Surprisingly, AICDA expression was induced by ultraviolet B (UVB) irradiation and further increased by a combination of UVB and IL-1 $\beta$ in human cutaneous SCC (HSC1) and melanoma (G361) cell lines (Figure $5 \mathrm{~B}$ ). We found that single-agent treatment with Poly I:C, which consists of dsRNA, could induce AICDA expression. These results indicate that endogenous AID can be expressed in keratinocytes in a stimulus-dependent manner. 
Table 3. Mutation frequency of Trp53 in TPA-alone-treated epidermis from AID-deficient mice and littermate FVB/N WT mice

\begin{tabular}{|c|c|c|c|c|c|}
\hline Gene & Mouse & Tissue & Mutated/total clones & Mutated/total bases & Mutation frequency $\left(\times 10^{-4}\right)$ \\
\hline $\operatorname{Trp53}$ & FVB/N WT & Epidermis (TPA alone) & $10 / 78$ & $10 / 66,768$ & $1.50^{\mathrm{A}}$ \\
\hline & $\mathrm{AlD}^{-1-} \mathrm{FVB} / \mathrm{N}$ & Epidermis (TPA alone) & $3 / 80$ & $3 / 69,280$ & 0.43 \\
\hline
\end{tabular}

Dorsal skin of the mice was treated with $16 \mathrm{nmol}$ TPA twice weekly for 21 weeks, and $\operatorname{Trp} 53$ mutation was analyzed in the epidermis. Trp53 transcripts were amplified, and the PCR products were cloned into a vector and sequenced. ${ }^{A} P<0.05$, by $\chi^{2}$ test. A detailed list of mutations is provided in Supplemental Figure 5.

To further substantiate TPA-induced AID expression, we used a genetic lineage-tracing approach (32) to track cells that expressed or had previously expressed AID in the epidermis. Aicda-Cre and $R 26 R$ double-Tg mice (Aicda-Cre R26R), in which AID promoterdriven Cre recombinase irreversibly induces lacZ expression, were used to identify cells with a history of AID expression (Figure 6A and refs. 33, 34). Aicda-Cre is a line of bacterial artificial chromosome (BAC) Tg mice that harbor the whole Aicda genomic locus with a targeted insertion of the Cre-coding sequence (35). R26R is a Cre reporter strain in which a loxP-flanked neomycin gene with a transcriptional terminator and lacZ gene are inserted into the ubiquitously expressed Gt(ROSA)26Sor locus (36). In agreement with the known distribution of AID expression, lacZ expression was observed in germinal center B cells in Peyer's patches from AicdaCre R26R mice, but was rarely detectable in the thymus (Figure 6, $\mathrm{B}-\mathrm{D})$. Although gross examination of nontreated skin from AicdaCre $R 26 R$ mice revealed little lacZ staining (Figure 6E), microscopic analysis revealed very specific lac $Z$ expression patterns confined to the root sheaths adjacent to sebaceous glands and dermal papilla located at hair bulbs (Figure 6, F and G). This AID activity localization has not previously been reported, and its physiological significance requires further study. In the TPA-treated skin of Aicda-Cre R26R mice, intense lacZ staining was observed around the hair follicle upon gross examination (Figure $6 \mathrm{H}$ ), and microscopic analysis revealed distinct lacZ-positive cell populations in focal areas of the interfollicular epidermis, inner and outer root sheath, dermal papilla, and hair bulb matrix (Figure 6, I-M). In addition, we identified sporadic lacZ staining in fibroblastlike cells in the dermis and muscular layers (Figure 6I). In TPAtreated skin of $R 26 R$ single-Tg mice, weak and diffuse background lacZ staining because of endogenous $\beta$-gal activity was observed, whereas staining was absent in the hair follicles or interfollicular epidermis (Figure 6, N-P). These findings indicate that AID is physiologically expressed in parts of the hair follicle. Under TPAstimulated conditions, AID-expressing cells migrated into the interfollicular epidermis, or newly generated AID-expressing cells emerged from the epidermis.

To investigate whether stimulus-triggered endogenous AID expression could introduce mutation, skin samples from AIDdeficient mice and littermate FVB/N WT mice were treated with TPA alone twice weekly for 21 weeks, and the epidermis was analyzed for Trp53 mutation. We identified Trp53 gene mutations in WT mice and a significantly higher mutation frequency in WT mice than in AID-deficient mice (Table 3 and Supplemental Figure $5 \mathrm{~A}$ ). Notably, $60 \%$ (6 of 10 ) of base substitutions in WT mice were C-to-T transitions, in which $67 \%$ (4 of 6) of mutations were 5'-flanked by G (Supplemental Figure 5B). This pattern was consistent with the mutational signature preferred by AID. These findings suggest that chronic epidermal stimulation using TPA results in an accumulation of Trp53 mutations via upregulation of endogenous AID, albeit at a low frequency.

AID is expressed in human skin cancers and in histologically related head and neck cancers. To confirm whether AID is expressed in human skin cancers, we examined its expression in clinical samples of skin cancer, head and neck cancer, and related precancerous lesions (Figure 7A and Supplemental Table 6). Following antiAID immunostaining, we detected AID-positive cells in human cutaneous SCC, BCC, melanoma, and precancerous lesions of Bowen's disease and actinic keratosis. Interestingly, psoriasis, an inflammatory skin disorder with acanthosis, was positive for AID. Furthermore, we detected AID-positive cells in human head and neck SCC (HNSCC), melanoma, and related precancerous lesions of leukoplakia and lichen planus. In addition, we examined AICDA transcript levels in frozen samples of human skin cancer and head and neck cancer (Figure 7B). AICDA transcripts were detected in SCC, BCC, and melanoma, and their expression levels were at most approximately $10 \%$ of those in a Burkitt's lymphoma cell line, BL2. To clarify whether AICDA transcripts were derived from $B$ lymphocytes that had infiltrated tissues, we examined the samples for CD19 expression (Figure 7B). CD19 expression did not correlate with AICDA expression, thus excluding the possibility of B lymphocyte contamination in the tissues.

We performed a statistical analysis to determine whether AID expression in human skin cancer and head and neck cancer was associated with patients' clinical characteristics and demographics (Table 4 ). Overall, $42.5 \%$ of skin cancers and $44.4 \%$ of head and neck cancers, including precancers, were AID positive. According to histological type, the AID positivity rates were $33.3 \%$ (SCC), $15.4 \%$ (BCC), and $42.9 \%$ (melanoma) among skin cancers and $40.0 \%$ (SCC) and $25.0 \%$ (melanoma) among head and neck cancers. Patients' sex and age were not associated with AID expression levels. Skin specimens with cancers thought to have resulted from UV exposure tended to be AID positive, although this finding was not significant $(P=0.062 ; 95 \% \mathrm{CI}, 0.980-14.226)$. Among head and neck cancers, early tumor, node, metastasisstage (TNM-stage) disease (stage 0, I, or II) showed a significantly stronger association with AID expression than did late-stage disease (stage III or IV; $P=0.047$; 95\% CI, 0.998-125.644). Tobacco and alcohol use, which are the major causes of head and neck cancer, were not associated with AID expression. Preoperative neoadjuvant chemotherapy was significantly associated with impaired AID expression in HNSCC $(P=0.049)$. 
A
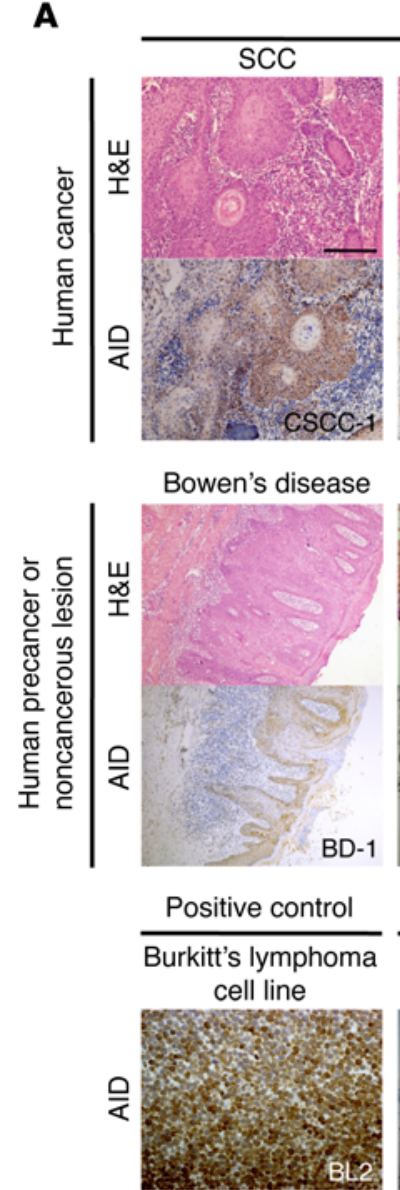

Cutaneous

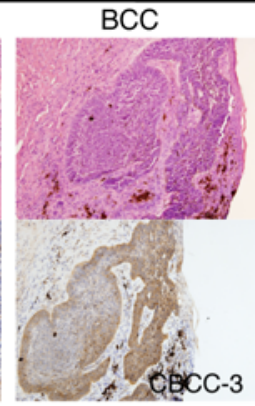

Actinic keratosis

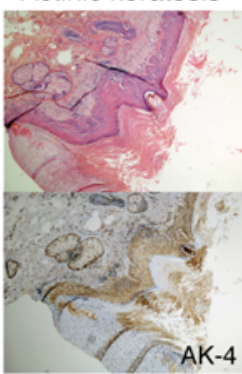

Negative control

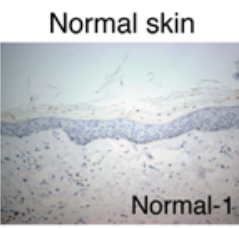

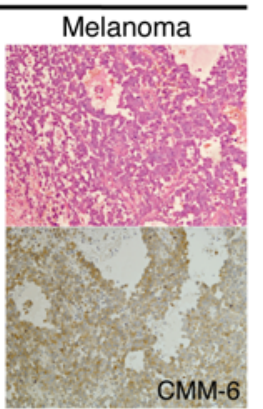

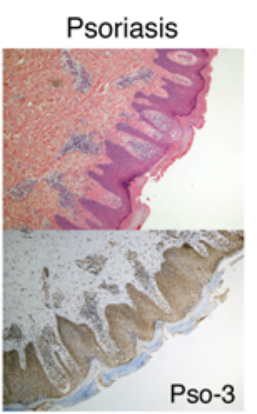

Pso-3

\section{B}

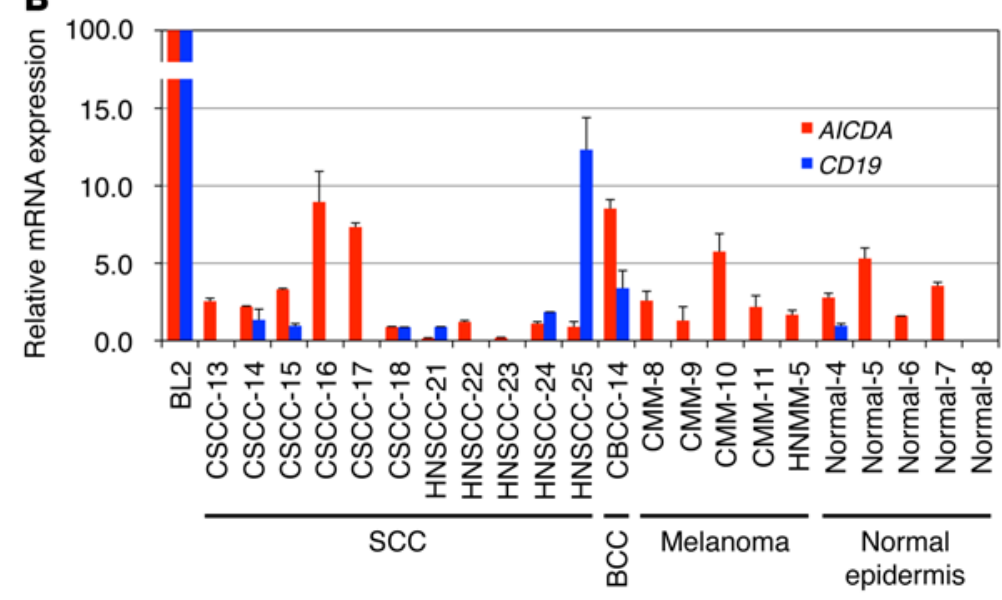

Figure 7. AID expression in human skin cancer and head and neck cancer. (A) Representative photomicrographs of human skin cancer, head and neck cancer, precancer, and noncancerous lesions showing AID expression. CSCC, cutaneous SCC; CBCC, cutaneous BCC; CMM cutaneous malignant melanoma; HNMM, head and neck malignant melanoma; BD, Bowen's disease; AK, actinic keratosis; Pso, psoriasis; Leu, leukoplakia; LP, lichen planus; BL2, Burkitt's Iymphoma cell line. Scale bar: $200 \mu \mathrm{m}$. Identification numbers correspond to the list of patients in Supplemental Table 6. (B) Relative AICDA and CD19 mRNA expression in human skin cancer and head and neck cancer (mean $\pm S D, n=3$ ).

TP53 mutations in human cutaneous SCC in the UV-protected regions show predominant $C$-to-T transition. Clothing is the first line of defense against the sun's UV rays, and therefore genital skin is among the areas most protected against UV light on the human body. Most skin cancers of the male and female external genitalia are therefore attributed to reasons other than UV light. However, the precise pathogenic mechanism is not clear, except for cases involving human papilloma virus (HPV) (37). The current version of the IARC TP53 mutation database (22) contains a total of 88 cases of cutaneous SCC of the male and female external genitalia. To investigate the TP53 mutation patterns in cutaneous SCC of sun-protected regions, 45 cases without HPV infection, radiation treatment, or hereditary predisposition were selected for analysis. Analysis of non-UV-induced TP53 mutations in these external genitalia SCCs revealed that $95.6 \%$ (43 of 45 ) of TP53 mutations were concentrated in the DNA-binding domain (Supplemental Figure $6 \mathrm{~A}$ ), and $67.4 \%$ (29 of 43 ) of the base substitutions comprised C-to-T transitions, in which 48\% (14 of 29) of mutations were 5'-flanked by G or A (Supplemental Figure 6B). These results indicate that the TP53 mutation pattern in non-sun-exposed regions is consistent with the pattern induced by AID activity.

\section{Discussion}

Here, we have described the contributions of AID in a mouse model of skin cancer and considered its implications in human skin cancer. Tumor incidence was increased in a mouse model of chemically induced skin cancer in response to Tg AID expression; however, it was decreased by Aicda gene disruption. Moreover, 
Table 4. Baseline characteristics of patients with skin cancer and head and neck cancer, according to AID expression status

\section{Characteristic}

Human skin cancer

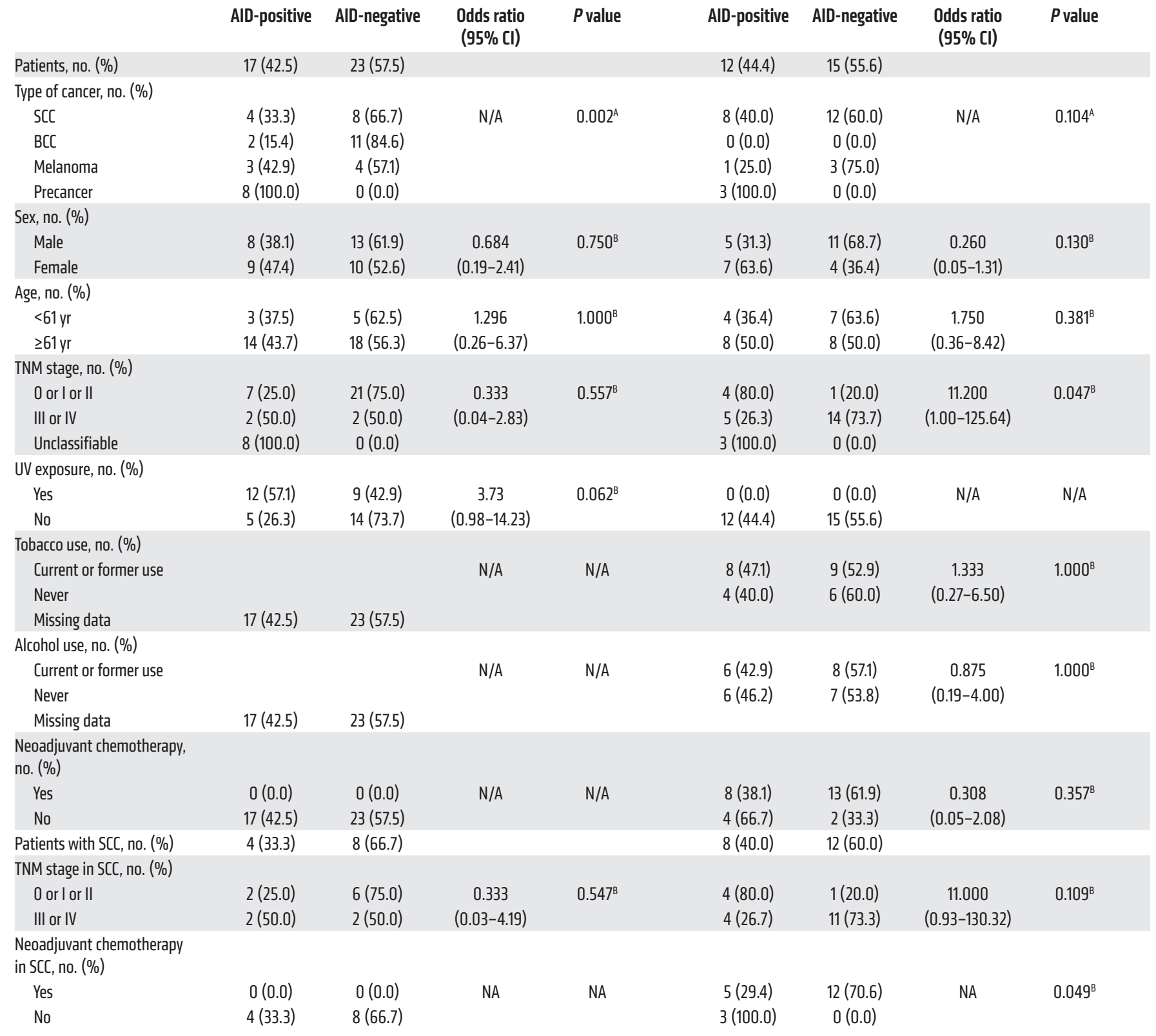

${ }^{A} P$ value was calculated using the $\chi^{2}$ test. ${ }^{B} P$ value was calculated using Fisher's exact test. A detailed list of patients' clinical characteristics is provided in Supplemental Table 6.

AID-Tg mice spontaneously developed skin tumors in the absence of carcinogen treatment, the incidence of which increased dramatically in immunodeficient nude mice compared with that observed in immunocompetent mice. Hras1 mutations, including Q61L, which is induced by a chemical mutagen (DMBA), were observed in skin cancer tissues from AID-Tg mice without DMBA. Trp53 mutations were identified in a pattern consistent with a mutational signature preferred by AID. We found that AID expression could be induced in human keratinocytes by several stimuli, including inflammatory cytokines (TGF- $\beta$ and IL-1 $\beta$ ), bacterial antigens (LPS), and UV irradiation. Furthermore, several types of human skin cancer and head and neck cancer expressed AID, suggesting the role of AID during carcinogenesis in human stratified squamous epithelium.

The chemically induced murine skin tumor model represents a critical step in the history of cancer research, because it provided the first indication that cancer occurs through a multistep process. Similar rodent tumor models that incorporate DNA mutagens have been developed, including the induction of oral epithelial cancer by $4 \mathrm{NQO}$ (16) and small intestinal tumors by azoxymethane (AOM) and dextran sodium sulfate (DSS) (38). Using a skin cancer model based on our AID-Tg mice, we demonstrated that 


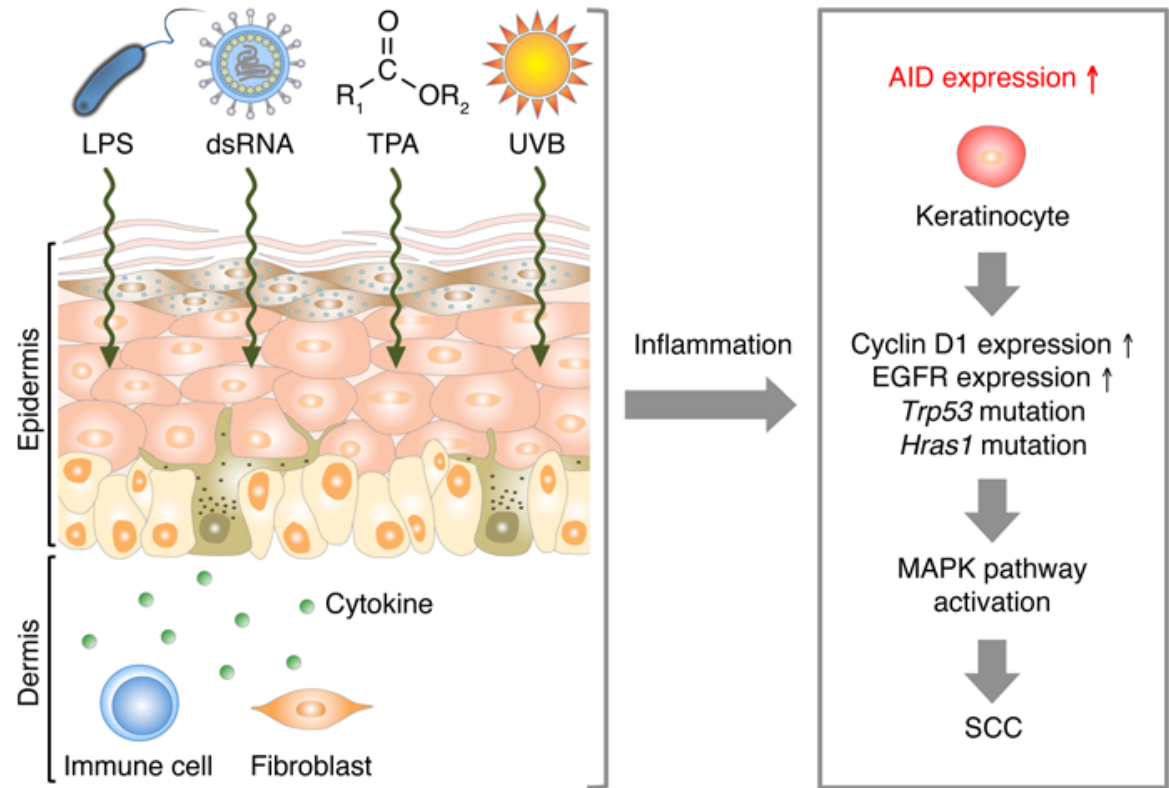

Figure 8. Working model of skin carcinogenesis mediated by inflammation-induced AID expression. LPS, dsRNA, TPA, and UVB from the outside of the epidermis, and inflammatory cytokines from immune cells and fibroblasts increase AID expression in keratinocytes. AID increases cyclin D1 and EGFR expression and introduces mutations in Trp53 and Hras1 genes to activate the MAPK pathway. Repetitive stimulation and chronic inflammation maintain upregulation of AID, causing an accumulation of mutations in Trp53 and Hras1 genes and thereby promoting malignant transformation. Upward arrow indicates upregulation.
Tg AID acts as a mutagen, thus substituting for the DMBA requirement and synergizing with TPA. Unexpectedly, in a mirror experiment wherein TPA was omitted, AID synergized with DMBA, although the effect was not as strong as that observed with TPA. How does AID exert this tumor promoter-like activity?

Tumor promotion is traditionally defined as an effect that causes clonal expansion of initiated cells. AID could stimulate cell division through at least 2 possible mechanisms. One hypothesis states that AID perturbs the DNA methylation status and epigenetic regulation of gene expression. Previous studies have shown that AID is required for OCT4 and NANOG promoter demethylation in somatic cells (39) and for genome-wide DNA demethylation in primordial germ cells (40). If AID causes DNA demethylation in the promoter regions of oncogenes, the consequent transcriptional upregulation may stimulate cell proliferation (41). Another explanation for AID-mediated cell division is compensatory proliferation after cell injury. Recently, we reported that CAG promoter-driven AID-Tg mice developed peculiar structures in the lung alveoli, which express lung regeneration markers such as p63, keratins 5 and 14, and LGR5/6 (42). Increased apoptosis in the lungs and livers of AID-Tg mice, with reported AID cytotoxicity in cultured cells (43), led us to speculate that AID may indirectly stimulate cell division in vivo as a physiological response to AID-induced tissue damage. A similar compensatory proliferation that predisposes mice to liver cancer was previously reported (44). This mechanism of cell proliferation may explain the weak tumor-promoting effect of the K14-AID transgene. Tissue injury involvement is suggested because of the distribution of spontaneously developed skin tumors in K14-AID-Tg mice, which were concentrated in the head and neck regions where an association with scratching could be suspected (Supplemental Table 1).

A previous study showed that long-term treatment with TPA alone resulted in SCC development of BALB/c mice, suggesting that TPA is a weak carcinogen (45). That earlier finding may be explained by our finding that TPA induced AID expression in keratinocytes. Therefore, the action of TPA may involve not only PKC- driven cell proliferation, but also AID-mediated mutagenesis. The percentage of papillomas that progressed to SCC was significantly higher in AID-Tg mice than in their WT littermates. Our findings, and those of a previous study that demonstrated enhanced malignant progression of chemically induced skin tumors in p53-KO mice (46), are compatible with a view in which genomic instability accelerates tumor progression to a more malignant state (47). A recent whole-exome sequence analysis of DMBA-plus-TPAinduced skin SCC in mice revealed that the pattern of genetic changes during progression from papilloma to SCC is distinct from the DMBA signature (25). TPA-induced AID expression may underlie genetic changes during progression to SCC.

Because the keratin 14 promoter is active in thymic epithelium, the K14-AID transgene may perturb $\mathrm{T}$ cell development, the dysregulation of which may be a major contributor to frequent skin tumor development. However, tumor enhancement by the K14-AID transgene on a nude mouse background, which lacks $\mathrm{T}$ cells (Figure 1 and Supplemental Figure 2), contradicts this possibility. Augmented skin tumor incidence was reported in carcinogen- or UV light-treated nude mice without the AID transgene (48). This and our studies may reflect effective T cell-mediated immunity against murine skin tumors.

Next, we will discuss the potential involvement of endogenous AID in murine tumor models. We previously reported that endogenous AID expression was required for malignant tumor progression in a mouse model of intestinal cancer by comparing tumor frequencies between AID-sufficient and -deficient mice that both harbored deficiencies in a gene encoding the immunosuppressive cytokine IL-10 (49). In the present study, we performed a similar experiment using a skin cancer model and reached the same conclusion: AID deficiency decreases tumor incidence. Furthermore, we repeated this experiment in the absence of $\mathrm{B}$ lymphocytes to eliminate indirect effects from a lack of $\mathrm{Ab}$ diversification by class switching and somatic hypermutation, both of which are triggered by AID. Even this strict comparison between the presence and absence of AID activity in non-B cells supported our conclusion. 
Although the AID expression level is lower in keratinocytes than in B cells, AID definitively influences tumor incidence and likely does so by increasing the mutational frequency.

A previous report demonstrated AID expression in a restricted population of T cells (33). It is difficult for us to formally exclude the possibility that AID expression in T cells is required for DMBAplus-TPA-induced skin tumors. A formal test of the involvement of AID in keratinocytes during skin tumor formation would require a mouse strain with a keratinocyte-specific Aicda gene KO, which is unavailable at present.

In the present study, the main Trp53 mutation pattern observed in the tumor samples (Figure 4D) and nontumor epidermal samples stimulated by TPA (Supplemental Figure 5B) was a $\mathrm{C} / \mathrm{G}$ to $\mathrm{T} / \mathrm{A}$ transition with mutational signatures compatible with that of AID. In contrast, the mutations observed in Hras1 did not resemble the cytidine deaminase footprint. Hras1 mutations appeared to emerge as a result of vigorous positive selection that favored its constitutive activation. AID can cause mutations other than the $\mathrm{C} / \mathrm{G}$ to $\mathrm{T} / \mathrm{A}$ transition through indirect mechanisms involving DNA mismatch repair $(50,51)$ and topoisomerase $1(52)$. Therefore, it is not surprising that G-to-T or A-to-T mutations are caused by AID, albeit at a low frequency.

Cancer diversity is recognized not only in the mutated gene repertoire but also in a variety of mutational mechanisms. Recently, a genome-wide mutation analysis of human cancers identified mutational signatures compatible with mutations caused by AID and APOBEC family members (53). However, it may be difficult to assess the contribution of each cytidine deaminase in individual cases of human cancer. It has been postulated that AID acts on transcribed single-stranded DNA and preferentially mutates cytidines in the WRC motif (W is A or T; R is A or G) $(26,54,55)$. On the other hand, $\mathrm{T}$ or $\mathrm{C}$ is often the $5^{\prime}$-flanking nucleotide of APOBEC3s-induced C-to-T transitions (27). We found that when the skin of FVB/N WT mice was treated with TPA alone, $67 \%$ of C-to-T transitions in the Trp53 gene were preceded by $\mathrm{G}$ (Supplemental Figure $5 \mathrm{~B}$ ). The same signature was observed in Trp53 gene mutations from the spontaneous SCC from K14-AID-Tg mice (Figure 4D). These results suggest that some C-to-T transitions are caused by AID, although it may be impossible to determine the responsible enzymes for each mutation solely on the basis of the 5'-flanking nucleotide.

A particularly intriguing finding is that AID expression is induced by UVB irradiation in human keratinocytes. Among the TP53 gene mutations identified in human nonmelanoma skin cancers, C-to-T transitions located at dipyrimidine sites (UV signature mutation) are common and more frequent than those observed in internal organ cancers (21). Nonetheless, some mutation patterns cannot be explained by UV-induced mutational signatures. These patterns may instead be explained by AID-induced DNA damage or oxidative DNA damage via the production of 8-hydroxydeoxyguanosine by UVB irradiation (56). A previous study showed that UVB irradiation promotes skin tumor formation in mice treated with DMBA alone, suggesting that UV light can act as a tumor promoter and synergize with DMBA (57). The role of UV light in facilitating skin tumor formation may include not only its direct DNA damage but also indirect damage via AID, in addition to AID-independent cytotoxicity resulting in compensatory cell division, as discussed for AID.
Whereas skin cancer on non-sun-exposed areas is not very common in lighter-skinned people (i.e., those of mixed European ancestry), it is common in people of color, which traditionally refers to those of Asian, African, Middle Eastern, Hispanic, and Native American descent (9). It has been proposed that the $\mathrm{p} 53$ mutations identified in skin cancers occurring in sun-protected areas are caused by poorly characterized non-UV factors. Our analysis of the IARC TP53 database confirmed a predominant C-to-T transition in human cutaneous SCC of UV-protected regions such as the penis and vulva, supporting the hypothesis that C-to-T transitions in the non-sun-exposed areas are caused by cytidine deaminases. Half of these C-to-T transitions were 5 '-flanked by G or A, the preferential target of AID.

A case of human HNSCC with AID expression was previously reported (58). Another group reported AID positivity in $37.0 \%$ of human HNSCC cases, with early-stage cancers more frequently expressing AID than late-stage cancers (59). Our results from human HNSCC samples, together with these previous results, support the role for AID in the development of human HNSCC.

Remarkably, all human precancers examined in this study exhibited positive AID staining (1 case of Bowen's disease, 7 of actinic keratoses, 2 of leukoplakia, and 1 of lichen planus), a 3 -fold higher rate than the average rate among cancers, although the number of samples was small. A high rate of TP53 mutation was reported in lichen planus (60), consistent with the general notion that precancerous lesions have already accumulated somatic mutations. The fitness of cancer cells may be compromised because of excessive mutations if AID expression continues after full malignancy ("Muller's ratchet;" ref. 61). Therefore, established cancers might downregulate AID expression to avoid harmful mutations.

Furthermore, we examined 5 cases of human psoriasis, all of which were found to be AID positive. Psoriasis is an inflammatory keratosis of unknown etiology without a cancer predisposition. A strong inflammatory milieu may be responsible for AID induction in keratinocytes. The development of psoriatic lesions was observed in CAG-AID-Tg mice on a nude background, but not in those on a normal C57BL/6 background. Further studies should address whether AID expression is a driver of psoriatic changes or merely a consequence of dermal inflammation.

What is the physiological role of AID expressed in the epidermis and head and neck mucosa? Intriguingly, fate mapping demonstrated a unique localization pattern of AID in hair follicles. One possible explanation is that this phenomenon may be part of an antiviral defense mechanism. APOBEC3 proteins play a role in innate immunity by deaminating cytosines in viral and retrotransposon genomes (62). Similarly, AID induction by Poly I:C in keratinocytes and melanocytes may play an as-yet uncovered antiviral role.

Our present findings demonstrate that the mutagenic enzyme AID responds to stimuli originating from both outside and inside the epidermis (Figure 8). Various external stimuli such as bacterial LPS and viral dsRNA, UVB, and TPA can induce AID expression. On the other hand, cytokines released from dermal cells stimulate keratinocytes to express AID. If induced AID expression in keratinocytes contributes to carcinogenesis, therapeutic strategies targeting AID may effectively prevent skin cancer and head and neck cancer. 


\section{Methods}

Mice. CAG-AID-Tg mice were described previously (3). CAG-AID$\mathrm{Tg}$ mice were backcrossed with nude mice (KSN Slc-nu/nu) over 5 generations to generate CAG-AID-Tg nu/nu mice. Nude mice (KSN Slc-nu/nu) were purchased from Shimizu Laboratory Supplies. The pG3Z-K14 cassette (a gift of Akira Kakizuka, Kyoto University, Kyoto, Japan) (63) was used to construct the K14-AID transgene. Murine Aicda cDNA was purified as a blunt-ended EcoRI-BamHI fragment from the pFB-mAID-IRES-GFP vector (64) and ligated into the blunt-ended BamHI-digested pG3Z-K14 cassette. The purified EcoRI-HindIII fragment containing the K14-AID transgene was microinjected into fertilized FVB/N eggs to generate K14-AID-Tg mice. AID-deficient mice $(\mathrm{C} 57 \mathrm{BL} / 6)$ were described previously (2) and were backcrossed with FVB/N mice over 5 generations. K14-AID ${ }^{\text {hi }}$-Tg mice were backcrossed with nude mice (BALB/cA Jcl nu/nu) over 5 generations to generate K14-AID ${ }^{\text {hi }} \mathrm{nu} / \mathrm{nu}$ mice. The AID-deficient and K14-AID ${ }^{\mathrm{hi}} \mathrm{nu} / \mathrm{nu}$ mice, which bear homozygous Ptch 1 T1267N alleles, were selected by screening as described in the next section and used for experiments. The AID-deficient (C57BL/6) mice were backcrossed with $\mu \mathrm{MT}$ (C57BL/6) mice to generate AID-deficient $\mu \mathrm{MT}$ mice. FVB/N mice and nude (BALB/cA Jcl-nu/nu) mice were purchased from CLEA Japan, and $\mu \mathrm{MT}$ (B6.129S2-Igh-6<tm1Cgn $>/ \mathrm{J}$ ) mice were purchased from The Jackson Laboratory. Aicda-Cre mice (35) were a gift of Meinrad Busslinger (Research Institute of Molecular Pathology, Vienna, Austria). $R 26 R$ mice (36) were purchased from The Jackson Laboratory [B6;129S4-Gt(ROSA)26Sor $\left.{ }^{\text {tm1Sor }} / \mathrm{J}\right]$. Aicda-Cre mice were crossed with $R 26 R$ mice to generate double-Tg mice, in which cells with a history of AID expression constitutively express the lacZ gene.

Ptch1 T1267N SNP genotyping. The single allele-specific TaqMan MGB probe and primer sets for the Ptch1 gene were designed using Primer Express Software (Applied Biosystems) and Oligo Primer Analysis Software (Molecular Biology Insights). Mice possessing the T1267N polymorphism in Ptch1 were screened by an allelic discrimination assay, using genomic DNA from tail biopsies and a real-time thermal cycler (Mx3000P; Stratagene). The primer and TaqMan MGB probe sequences are shown in Supplemental Table 5.

Chemically induced skin carcinogenesis. A multistage chemically induced skin carcinogenesis experiment was performed using DMBA and TPA as described previously (13). A single 260-nmol dose of DMBA (D3254; Sigma-Aldrich) was applied topically to the shaved dorsal skin of mice. Two weeks after initiation, a 16-nmol dose of TPA (P8139; Sigma-Aldrich) was applied twice weekly to the skin for the duration of the experiment.

Chemically induced oral carcinogenesis. The $4 \mathrm{NQO}$-induced oral carcinogenesis experiment was performed as described previously (65). Murine oral mucosa was treated with $20 \mu \mathrm{l} 4 \mathrm{NQO}$ (Wako) dissolved in propylene glycol $(5 \mathrm{mg} / \mathrm{ml})$ thrice weekly.

Mutation analysis. Total RNA was isolated from tissues using TRIzol Reagent (Life Technologies) and an RNeasy Mini Kit (QIAGEN); cDNA was subsequently synthesized using the iScript cDNA Synthesis Kit (Bio-Rad). PCR fragments were generated using Phusion Highfidelity DNA Polymerase (Finnzymes, Thermo Fisher Scientific). PCR products were directly sequenced, except those from tumors in CAG-AID-Tg mice and nontumor epidermis stimulated by TPA alone, which were subcloned using the Zero Blunt TOPO PCR Cloning Kit for Sequencing (Invitrogen) and sequenced. Sequences were compared with those in the Ensembl Genome Browser database (http://www. ensembl.org/index.html) for ENSMUST00000026572 (Hras1) and ENSMUST00000005371 (Trp53). Amino acid sequences of mouse p53 in SCC from K14-AID-Tg mice were compared with those in human p53. Data for TP53 somatic mutations in skin SCC were downloaded from the IARC TP53 database (version R16, November 2012) (22). Primer and probe sequences are shown in Supplemental Table 5.

IHC. Formalin-fixed, paraffin-embedded (FFPE) mouse tissue sections were stained using an avidin/biotin system with rat monoclonal anti-mouse AID Ab MAID-2 (66); rabbit polyclonal anti-cyclin D1 Ab (C-20; Santa Cruz Biotechnology Inc.); rabbit polyclonal antiEGFR Ab (sc-1005; Santa Cruz Biotechnology Inc.); rabbit monoclonal anti-phospho-p44/42 MAPK Ab (D13.14.4E, Thr202/Tyr204; Cell Signaling Technology); rabbit monoclonal anti-phospho-AKT $\mathrm{Ab}$ (D9E, Ser473; Cell Signaling Technology); and mouse monoclonal anti-p16 Ab (F-12; Santa Cruz Biotechnology Inc.). FFPE human tissue sections were stained using the avidin/biotin system with a mouse polyclonal anti-human AID Ab (67). Endogenous epidermal AID expression 48 hours after treatment with $16 \mathrm{nmol}$ TPA was detected using a tyramide signal amplification system with TSA Plus TMR kits (PerkinElmer) (68). Images were obtained using a DM5000B microscope (Leica Microsystems).

AID fate mapping. TPA (16 nmol) was applied twice weekly to the shaved dorsal skin of 6-week-old R26R single-Tg mice and 6-weekold Aicda-Cre and R26R transgene double-Tg mice. After 6 weeks of treatment (13 doses), mice were anesthetized via an i.p. injection of 10 mg pentobarbital sodium salt and perfused from the left ventricle with fixative solution (2.2\% formaldehyde, $0.2 \%$ glutaraldehyde, $1 \times \mathrm{PBS}$ ). Dorsal skin was resected and further fixed with the same solution for 30 minutes. Fixed skin was washed with $1 \times$ PBS for 30 minutes and with a detergent rinse $(0.02 \%$ Nonidet $\mathrm{P}-40,0.2 \mathrm{M}$ sodium phosphate, pH 7.0, 0.01\% sodium deoxycholate) for 30 minutes. Skin was developed using staining buffer $(1 \mathrm{mg} / \mathrm{ml} \mathrm{X}$-gal, $1 \mathrm{mM}$ magnesium chloride, $3 \mathrm{mM}$ potassium ferrocyanide, $3 \mathrm{mM}$ potassium ferricyanide, $0.1 \%$ Triton $\mathrm{X}$ ) at $37^{\circ} \mathrm{C}$ for 20 hours. After macroscopic observation, skin was dehydrated in ethanol and embedded in paraffin. Deparaffinized 10 - $\mu \mathrm{m}$-thick sections were observed under a microscope.

Cell culture. Primary human adult keratinocytes were purchased from Kurabo and grown in HuMedia-KB2 serum-free medium (Kurabo). The human cutaneous SCC cell line HSC1 was purchased from the Japan Health Sciences Foundation (cell bank registration JCRB1015) and cultured in DMEM with 20\% FBS. The human skin malignant melanoma cell line G361 was purchased from the Japan Health Sciences Foundation (cell bank registration JCRB9074) and cultured in McCoy's 5A medium with $10 \%$ FBS. Cells were stimulated in culture medium supplemented with $75 \mu \mathrm{g} / \mathrm{ml}$ LPS (L3137; Sigma-Aldrich); $100 \mu \mathrm{g} / \mathrm{ml}$ Poly I:C (P9582; Sigma-Aldrich); 50 ng/ml PMA (P1585; Sigma-Aldrich); $1 \mu \mathrm{g} / \mathrm{ml}$ human CD40 ligand (C6362; Sigma-Aldrich); $10 \mathrm{ng} / \mathrm{ml}$ human IL-4 (I4269; SigmaAldrich); $10 \mathrm{ng} / \mathrm{ml}$ human TGF- $\beta$ (T7039; Sigma-Aldrich); $100 \mathrm{ng} / \mathrm{ml}$ human TNF- $\alpha$ (T0157; Sigma-Aldrich); and $25 \mathrm{ng} / \mathrm{ml}$ human IL-1 $\beta$ (200-01B; Peprotec). Cells were irradiated with $30 \mathrm{~mJ} / \mathrm{cm}^{2}$ of UVB using a UV irradiator (312 nm Bio-Link; Cosmo Bio).

$q R T-P C R$. Total RNA isolation and cDNA synthesis were performed as described in the Mutation analysis section. qRT-PCR was performed using QuantiTect Reagent (QIAGEN) and a real-time thermal cycler (Mx3000P; Stratagene). Gene expression levels were normalized to the expression levels of housekeeping genes (acidic 
ribosomal phosphoprotein PO [RPLPO] for humans and hypoxanthine guanine phosphoribosyl transferase [Hprt] for mice). Primer and probe sequences are shown in Supplemental Table 5.

Patients and controls. Frozen and FFPE clinical samples were obtained from 3 cohorts of individuals who underwent surgery or a biopsy procedure at the Department of Dermatology of the University of Occupational and Environmental Health, the Department of Dermatology of the Shiga Medical Center, and the Department of Otolaryngology Head and Neck Surgery of the Kyoto University. Clinical stages were determined according to the seventh edition of the American Joint Committee on Cancer (AJCC) staging system (69).

Statistics. All statistical analyses were performed using SPSS Statistics, version 21.0 (IBM), except the Kaplan-Meier analysis, which was performed using Prism 4.0 (GraphPad Software). Significant differences in mouse survival were assessed using the log-rank test. Welch's $t$ test was used for comparisons between 2 mouse groups of average tumor volumes and numbers. Pearson's correlation was calculated to estimate the statistical association between 2 quantitative gene expression variables. The $\chi^{2}$ test was used for mutation frequency analysis. Human cancer types were compared between AID expression groups using the $\chi^{2}$ test for categorical variables. Fisher's exact test was used to assess differences in the frequencies of categorical variables with a limited number of clinical samples. All statistical tests were 2 sided, and a $P$ value of less than 0.05 was considered statistically significant.

Study approval. All animal care and experiments were approved by the IACUCs of Kyoto University and Shiga Medical Center Research Institute. All work with human clinical specimens was approved by the Medical Ethics Committees of Kyoto University, Shiga Medical Center, and the University of Occupational and Envi- ronmental Health. Written informed consent was obtained from all patients prior to their inclusion in the study.

\section{Author contributions}

TN and KK designed the research studies, conducted experiments, acquired data, analyzed data, and wrote the manuscript. YT, HH, and MU conducted the experiments, acquired data, and analyzed the data. MN, NY, RA, YH, and KB provided essential materials and analyzed the data. NM analyzed the data.

\section{Acknowledgments}

We thank Yoko Kitawaki and Takae Toyoshima for technical assistance; Elaine Fuchs and Akira Kakizuka for providing the pG3Z-K14 cassette; Meinrad Busslinger for providing the AicdaCre mice; Il-mi Okazaki for providing the photograph of melanoma development in CAG-AID-Tg mice; Tasuku Honjo, Yoshiki Miyachi, and Juichi Ito for their initial support of this work; and Lewis L. Lanier for use of the laboratory facility. We also thank Hiroyuki Marusawa, Masamichi Muramatsu, Jiro Kitamura, Hiroyuki Nishimura, and Lewis L. Lanier for critically reading this manuscript. This study was supported by a Grant-in-Aid for Scientific Research (17013042, 18390122, and 23390097) and a Grant-in-Aid for JSPS Research Fellows from the Japan Society for the Promotion of Science, The Ichiro Kanehara Foundation, and The Kanae Foundation for the Promotion of Medical Science.

Address correspondence to: Kazuo Kinoshita, Shiga Medical Center Research Institute, 5-4-30 Moriyama, Shiga 524-8524, Japan. Phone: 81.77.582.6044; E-mail: kkinoshi.shigamed@mac.com.
1. Vogelstein B, Kinzler KW. Cancer genes and the pathways they control. Nat Med. 2004;10(8):789-799.

2. Muramatsu M, Kinoshita K, Fagarasan S, Yamada S, Shinkai Y, Honjo T. Class switch recombination and hypermutation require activation-induced cytidine deaminase (AID), a potential RNA editing enzyme. Cell. 2000;102(5):553-563.

3. Okazaki IM, et al. Constitutive expression of AID leads to tumorigenesis. JExp Med. 2003;197(9):1173-1181.

4. Takai A, et al. A novel mouse model of hepatocarcinogenesis triggered by AID causing deleterious p53 mutations. Oncogene. 2009;28(4):469-478.

5 . Kou T, et al. Expression of activation-induced cytidine deaminase in human hepatocytes during hepatocarcinogenesis. Int J Cancer. 2007;120(3):469-476.

6. Matsumoto Y, et al. Helicobacter pylori infection triggers aberrant expression of activationinduced cytidine deaminase in gastric epithelium. Nat Med. 2007;13(4):470-476.

7. Endo Y, et al. Activation-induced cytidine deaminase links between inflammation and the development of colitis-associated colorectal cancers. Gastroenterology. 2008;135(3):889-898.

8. Marusawa H. Aberrant AID expression and human cancer development. Int J Biochem Cell Biol. 2008;40(8):1399-1402.

9. Gloster HM Jr, Neal K. Skin cancer in skin of color. J Am Acad Dermatol. 2006;55(5):741-760.
10. Wakabayashi Y, Mao JH, Brown K, Girardi M, Balmain A. Promotion of Hras-induced squamous carcinomas by a polymorphic variant of the Patched gene in FVB mice. Nature. 2007;445(7129):761-765.

11. Muramatsu M, et al. Specific expression of activation-induced cytidine deaminase (AID), a novel member of the RNA-editing deaminase family in germinal center B cells. J Biol Chem. 1999;274(26):18470-18476.

12. Yuspa SH. The pathogenesis of squamous cell cancer: lessons learned from studies of skin carcinogenesis - thirty-third G. H. A. Clowes Memorial Award Lecture. Cancer Res. 1994;54(5):1178-1189.

13. Quintanilla M, Brown K, Ramsden M, Balmain A. Carcinogen-specific mutation and amplification of Ha-ras during mouse skin carcinogenesis. Nature. 1986;322(6074):78-80.

14. Andreu P, et al. FcR $\gamma$ activation regulates inflammation-associated squamous carcinogenesis. Cancer Cell. 2010;17(2):121-134.

15. Kitamura D, Roes J, Kuhn R, Rajewsky K. A B cell-deficient mouse by targeted disruption of the membrane exon of the immunoglobulin mu chain gene. Nature. 1991;350(6317):423-426.

16. Kanojia D, Vaidya MM. 4-nitroquinoline-1-oxide induced experimental oral carcinogenesis. Oral Oncol. 2006;42(7):655-667.

17. Tang XH, Knudsen B, Bemis D, Tickoo S, Gudas LJ. Oral cavity and esophageal carcinogenesis modeled in carcinogen-treated mice. Clin Cancer Res. 2004;10(1 pt 1):301-313.

18. Coleman ML, Marshall CJ, Olson MF. RAS and RHO GTPases in G1-phase cell-cycle regulation. Nat Rev Mol Cell Biol. 2004;5(5):355-366.

19. Karnoub AE, Weinberg RA. Ras oncogenes: split personalities. Nat Rev Mol Cell Biol. 2008;9(7):517-531.

20. Giglia-Mari G, Sarasin A. TP53 mutations in human skin cancers. Hum Mutat. 2003;21(3):217-228.

21. Ziegler A, et al. Sunburn and p53 in the onset of skin cancer. Nature. 1994;372(6508):773-776.

22. Petitjean A, et al. Impact of mutant p53 functional properties on TP53 mutation patterns and tumor phenotype: lessons from recent developments in the IARC TP53 database. Hum Mutat. 2007;28(6):622-629.

23. Luo JL, et al. UV-induced DNA damage and mutations in Hupki (human p53 knock-in) mice recapitulate 553 hotspot alterations in sun-exposed human skin. Cancer Res. 2001;61(22):8158-8163.

24. Kanjilal S, Pierceall WE, Cummings KK, Kripke ML, Ananthaswamy HN. High frequency of p53 mutations in ultraviolet radiation-induced murine skin tumors: evidence for strand bias and tumor heterogeneity. Cancer Res. 1993;53(13):2961-2964.

25. Nassar D, Latil M, Boeckx B, Lambrechts D, Blanpain C. Genomic landscape of carcinogen-induced and genetically induced mouse 
skin squamous cell carcinoma. Nat Med. 2015;21(8):946-954.

26. Larijani M, Martin A. Single-stranded DNA structure and positional context of the target cytidine determine the enzymatic efficiency of AID. $\mathrm{Mol}$ Cell Biol. 2007;27(23):8038-8048.

27. Henry M, Guetard D, Suspene R, Rusniok C, Wain-Hobson S, Vartanian JP. Genetic editing of HBV DNA by monodomain human APOBEC3 cytidine deaminases and the recombinant nature of APOBEC3G. PLoS One. 2009;4(1):e4277.

28. Uribe P, Gonzalez S. Epidermal growth factor receptor (EGFR) and squamous cell carcinoma of the skin: molecular bases for EGFR-targeted therapy. Pathol Res Pract. 2011;207(6):337-342.

29. Ruas M, Peters G. The p16INK4a/CDKN2A tumor suppressor and its relatives. Biochim Biophys Acta. 1998;1378(2):F115-F177.

30. Sharpless NE, DePinho RA. The INK4A/ARF locus and its two gene products. Curr Opin Genet Dev. 1999;9(1):22-30.

31. Matsumoto Y, Marusawa H, Kinoshita K, Niwa Y, Sakai Y, Chiba T. Up-regulation of activationinduced cytidine deaminase causes genetic aberrations at the CDKN2b-CDKN2a in gastric cancer. Gastroenterology. 2010;139(6):1984-1994.

32. Kretzschmar K, Watt FM. Lineage tracing. Cell. 2012;148(1-2):33-45.

33. Qin $\mathrm{H}$, et al. Activation-induced cytidine deaminase expression in $\mathrm{CD}^{+} \mathrm{T}$ cells is associated with a unique IL-10-producing subset that increases with age. PLoS One. 2011;6(12):e29141.

34. Rommel PC, et al. Fate mapping for activationinduced cytidine deaminase (AID) marks nonlymphoid cells during mouse development. PLOS One. 2013;8(7):e69208.

35 . Kwon K, et al. Instructive role of the transcription factor E2A in early B lymphopoiesis and germinal center B cell development. Immunity. 2008;28(6):751-762.

36. Soriano P. Generalized lacZ expression with the ROSA26 Cre reporter strain. Nat Genet. 1999;21(1):70-71.

37. Thomas LK, et al. Chromosomal gains and losses in human papillomavirus-associated neoplasia of the lower genital tract - a systematic review and meta-analysis. Eur J Cancer. 2014;50(1):85-98.

38. Whiteley LO, Hudson L Jr, Pretlow TP. Aberrant crypt foci in the colonic mucosa of rats treated with a genotoxic and nongenotoxic colon carcinogen. Toxicol Pathol. 1996;24(6):681-689.

39. Bhutani N, Brady JJ, Damian M, Sacco A, Corbel SY, Blau HM. Reprogramming towards pluripotency requires AID-dependent DNA demethylation. Nature. 2010;463(7284):1042-1047.

40. Popp C, et al. Genome-wide erasure of DNA methylation in mouse primordial germ cells is affected by AID deficiency. Nature.
2010;463(7284):1101-1105.

41. Isobe T, Song SN, Tiwari P, Ito H, Yamaguchi Y, Yoshizaki K. Activation-induced cytidine deaminase auto-activates and triggers aberrant gene expression. FEBS Lett. 2013;587(16):2487-2492.

42. Kitamura J, et al. Chronic lung injury by constitutive expression of activation-induced cytidine deaminase leads to focal mucous cell metaplasia and cancer. PLoS One. 2015;10(2):e0117986.

43. Hasham MG, et al. Widespread genomic breaks generated by activation-induced cytidine deaminase are prevented by homologous recombination. Nat Immunol. 2010;11(9):820-826.

44. Maeda S, Kamata H, Luo JL, Leffert H, Karin M. IKK $\beta$ couples hepatocyte death to cytokinedriven compensatory proliferation that promotes chemical hepatocarcinogenesis. Cell. 2005;121(7):977-990.

45. Astrup EG, Iversen OH. The tumourigenic and carcinogenic effect of 12-0-tetradecanoylphorbol-13-acetate when applied to the skin of BALB/cA and hairless (hr/hr) mice. Acta Pathol Microbiol Immunol Scand A. 1983;91(2):103-113.

46. Kemp CJ, Donehower LA, Bradley A, Balmain A. Reduction of $\mathrm{p} 53$ gene dosage does not increase initiation or promotion but enhances malignant progression of chemically induced skin tumors. Cell. 1993;74(5):813-822.

47. Morris SM. A role for p53 in the frequency and mechanism of mutation. Mutat Res. 2002;511(1):45-62.

48. Anderson LM, Rice JM. Tumorigenesis in athymic nude mouse skin by chemical carcinogens and ultraviolet light. J Natl Cancer Inst. 1987;78(1):125-134.

49. Takai A, et al. Targeting activation-induced cytidine deaminase prevents colon cancer development despite persistent colonic inflammation. Oncogene. 2012;31(13):1733-1742.

50. Di Noia JM, Neuberger MS. Molecular mechanisms of antibody somatic hypermutation. Annu Rev Biochem. 2007;76:1-22.

51. Peled JU, et al. The biochemistry of somatic hypermutation. Annu Rev Immunol. 2008;26:481-511.

52. Honjo T, Kobayashi M, Begum N, Kotani A, Sabouri S, Nagaoka H. The AID dilemma: infection, or cancer? Adv Cancer Res. 2012;113:1-44.

53. Alexandrov LB, et al. Signatures of mutational processes in human cancer. Nature. 2013;500(7463):415-421.

54. Pham P, Bransteitter R, Petruska J, Goodman MF. Processive AID-catalysed cytosine deamination on single-stranded DNA simulates somatic hypermutation. Nature. 2003;424(6944):103-107.

55. Yu K, Huang FT, Lieber MR. DNA substrate length and surrounding sequence affect the activation-induced deaminase activity at cytidine.
JBiol Chem. 2004;279(8):6496-6500.

56. Kato M, Iida M, Goto Y, Kondo T, Yajima I. Sunlight exposure-mediated DNA damage in young adults. Cancer Epidemiol Biomarkers Prev. 2011;20(8):1622-1628.

57. Epstein JH, Epstein WL. Cocarcinogenic effect of ultraviolet light on DMBA tumor initiation in albino mice. J Invest Dermatol. 1962;39(5):455-460.

58. Miyazaki Y, Inoue H, Kikuchi K, Ochiai K, Kusama K. Activation-induced cytidine deaminase mRNA expression in oral squamous cell carcinomaderived cell lines is upregulated by inflammatory cytokines. JOral Sci. 2012;54(1):71-75.

59. Nakanishi Y, et al. Role of activation-induced cytidine deaminase in the development of oral squamous cell carcinoma. PLoS One. 2013;8(4):e62066.

60. Ogmundsdottir HM, Hilmarsdottir H, Astvaldsdottir A, Johannsson JH, Holbrook WP. Oral lichen planus has a high rate of TP53 mutations. A study of oral mucosa in Iceland. Eur JOral Sci. 2002;110(3):192-198.

61. Muller HJ. Some genetic aspects of sex. American Naturalist. 1932;66(703):118-138.

62. Koito A, Ikeda T. Intrinsic immunity against retrotransposons by APOBEC cytidine deaminases. Front Microbiol. 2013;4:28.

63. Vassar R, Rosenberg M, Ross S, Tyner A, Fuchs E. Tissue-specific and differentiationspecific expression of a human K14 keratin gene in transgenic mice. Proc Natl Acad Sci U S A. 1989;86(5):1563-1567.

64. Begum NA, Kinoshita K, Muramatsu M, Nagaoka H, Shinkura R, Honjo T. De novo protein synthesis is required for activation-induced cytidine deaminase-dependent DNA cleavage in immunoglobulin class switch recombination. Proc Natl Acad Sci U S A . 2004;101(35):13003-13007.

65. Hawkins BL, Heniford BW, Ackermann DM, Leonberger M, Martinez SA, Hendler FJ. 4NQO carcinogenesis: a mouse model of oral cavity squamous cell carcinoma. Head Neck. 1994;16(5):424-432.

66. Tsuji M, et al. Requirement for lymphoid tissue-inducer cells in isolated follicle formation and $\mathrm{T}$ cell-independent immunoglobulin a generation in the gut. Immunity. 2008;29(2):261-271.

67. Ta VT, et al. AID mutant analyses indicate requirement for class-switch-specific cofactors. Nat Immunol. 2003;4(9):843-848.

68. Toda Y, et al. Application of tyramide signal amplification system to immunohistochemistry: a potent method to localize antigens that are not detectable by ordinary method. Pathol Int . 1999;49(5):479-483.

69. Edge SB, Byrd DR, Compton CC, Fritz AG, Greene FL, Trotti A. AJCC Cancer Staging Manual. 7th ed. New York, New York, USA: Springer; 2010. 